\title{
OSTEOCHONDRAL LESIONS IN DISTAL TARSAL JOINTS OF ICELANDIC HORSES REVEAL STRONG ASSOCIATIONS BETWEEN HYALINE AND CALCIFIED CARTILAGE ABNORMALITIES
}

\author{
C. J. Ley ${ }^{1, *}$, S. Ekman ${ }^{2}$, K. Hansson ${ }^{1}$, S. Björnsdóttir ${ }^{3}$ and A. Boyde ${ }^{4}$
}

\author{
${ }^{1}$ Department of Clinical Sciences, Swedish University of Agricultural Sciences, Uppsala, Sweden \\ ${ }^{2}$ Department of Biomedical Sciences and Veterinary Public Health, Swedish University of Agricultural Sciences, \\ Uppsala, Sweden \\ ${ }^{3}$ Icelandic Food and Veterinary Authority, Selfoss, Iceland \\ ${ }^{4}$ Biophysics, Oral Growth and Development, Dental Institute, Barts and The London School of Medicine and \\ Dentistry, Queen Mary University of London, UK
}

\begin{abstract}
Osteochondral lesions in the joints of the distal tarsal region of young Icelandic horses provide a natural model for the early stages of osteoarthritis (OA) in low-motion joints. We describe and characterise mineralised and non-mineralised osteochondral lesions in left distal tarsal region joint specimens from twenty-two $30 \pm 1$ month-old Icelandic horses. Combinations of confocal scanning light microscopy, backscattered electron scanning electron microscopy (including, importantly, iodine staining) and three-dimensional microcomputed tomography were used on specimens obtained with guidance from clinical imaging. Lesion-types were described and classified into groups according to morphological features. Their locations in the hyaline articular cartilage (HAC), articular calcified cartilage (ACC), subchondral bone (SCB) and the joint margin tissues were identified and their frequency in the joints recorded. Associations and correlations between lesion-types were investigated for centrodistal joints only. In centrodistal joints the lesion-types HAC chondrocyte loss, HAC fibrillation, HAC central chondrocyte clusters, ACC arrest and ACC advance had significant associations and strong correlations. These lesion-types had moderate to high frequency in centrodistal joints but low frequencies in tarsometatarsal and talocalcaneal-centroquartal joints. Joint margin lesion-types had no significant associations with other lesion-types in the centrodistal joints but high frequency in both the centrodistal and tarsometatarsal joints. The frequency of SCB lesion-types in all joints was low. Hypermineralised infill phase lesion-types were detected. Our results emphasise close associations between $\mathrm{HAC}$ and $\mathrm{ACC}$ lesions in equine centrodistal joints and the importance of ACC lesions in the development of OA in low-motion compression-loaded equine joints.
\end{abstract}

Keywords: Articular cartilage, subchondral bone, osteoarthritis, equine tarsus, backscattered electron microscopy, iodine-staining, confocal scanning light microscopy, microcomputed tomography.

Address for correspondence:

Charles J. Ley

Department of Clinical Sciences

Swedish University of Agricultural Sciences

Box 7054

SE-750 07 Uppsala, Sweden

\author{
Telephone Number: + 4618672013 \\ FAX Number: + 4618672811 \\ E-mail: charles.ley@slu.se
}

\section{Introduction}

The Icelandic horse provides an excellent model for the study of naturally occurring osteoarthritis $(\mathrm{OA})$ due to the heritability and high frequency of distal tarsal OA in the population (Eksell et al., 1998; Björnsdottir et al., 2000a; Björnsdottir et al., 2000b; Arnason and Björnsdóttir, 2003) and the relatively low genetic diversity of the breed (Glowatzki-Mullis et al., 2006). Although lameness does not usually develop until the horses are adult (Axelsson et al., 2001), it has been shown that distal tarsal OA lesions are seen with conventional light microscopy of paraffin-embedded specimens during the first year of age (Björnsdottir et al., 2004). Thus a study of the distal tarsal joints of young Icelandic horses provides an opportunity to study a spectrum of osteochondral lesions during the period when the early stages of OA are likely to be occurring.

Osteoarthritis is a disease that involves the entire joint organ (Waldschmidt et al., 1999; Guermazi et al., 2003; Peterfy et al., 2004), but in its early stages OA develops from focal microscopic regions (Dieppe and Kirwan, 1994; Squires et al., 2003; Veje et al., 2003; Pritzker et al., 2006) and controversy remains regarding which tissue type or types are affected in the earliest stages of OA.

Table 1. Abbreviations used in the text.

\begin{tabular}{|l|l|}
\hline Abbreviation & Definition \\
\hline 3D & Three-dimensional \\
\hline ACC & Articular calcified cartilage \\
\hline BSE SEM & $\begin{array}{l}\text { Backscattered electron scanning electron } \\
\text { microscopy }\end{array}$ \\
\hline CSLM & Confocal scanning light microscopy \\
\hline CT & Computed tomography \\
\hline HAC & Hyaline articular cartilage \\
\hline HIP & Hypermineralised infill phase \\
\hline MF & Mineralising front \\
\hline MRI & Magnetic resonance imaging \\
\hline OA & Osteoarthritis \\
\hline PMMA & Polymethyl methacrylate \\
\hline SCB & Subchondral bone \\
\hline$\mu$ CT & Microcomputed tomography \\
\hline
\end{tabular}




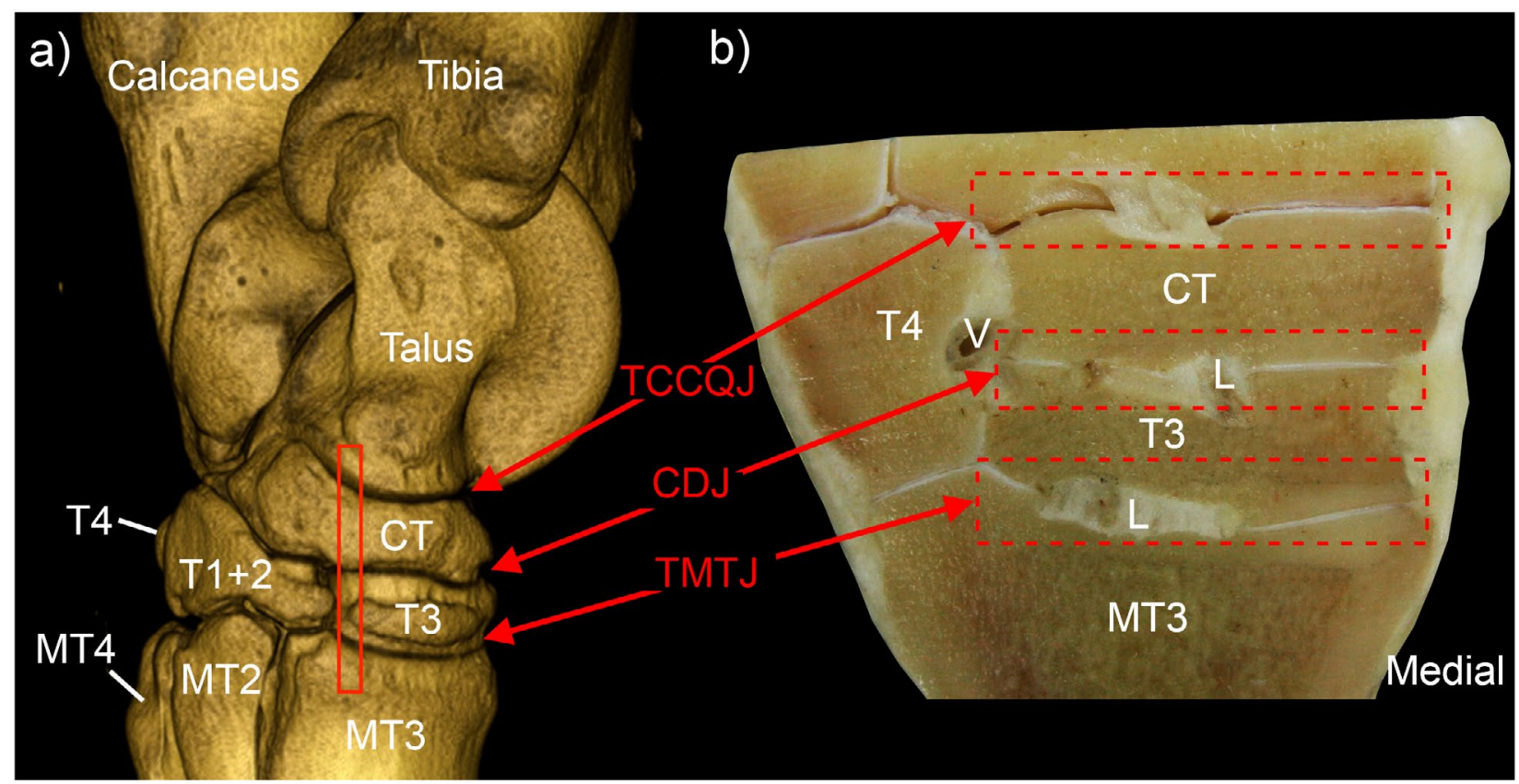

Fig. 1. Equine tarsal region anatomy. (a) Three-dimensional volumetric reconstruction CT image of the tarsal bones and joints, medial aspect left hind leg. (b) Photograph of the plantar surface of a frontal plane tarsal slab used in the study (location of origin shown by rectangle in a) with dashed line boxes marking the regions of the three joints used in the study. Talocalcaneal-centroquartal joint (TCCQJ); centrodistal joint (CDJ); tarsometatarsal joint (TMTJ); central tarsal bone (CT); fused first and second tarsal bones (T1+2); third tarsal bone (T3); fourth tarsal bone (T4); second metatarsal bone (MT2); third metatarsal bone (MT3); fourth metatarsal bone (MT4); interosseus ligaments (L); tarsea perforans artery and vein within the tarsal canal (V).

Proposals range from the articular cartilage (Björnsdottir et al., 2004; Heinegard and Saxne, 2011), the subchondral bone (SCB) (Brandt et al., 2006), the junction region of the articular cartilage and the bone (Suri and Walsh, 2012) and the synovium (Scanzello and Goldring, 2012). Thus, for efficient and accurate studies of early OA, methods are required that allow assessment of the entire joint organ, detect changes in the mineralised and non-mineralised tissues, and permit microscopic resolution. Recently a technique has been described that uses clinical magnetic resonance imaging (MRI) and computed tomography (CT) equipment to assess the entire joint and localise the position of suspected early focal osteochondral changes so that these areas can then be sampled for examination with microscopic techniques (Ley et al., 2013).

The major joints of the equine distal tarsal region are the talocalcaneal-centroquartal, centrodistal and tarsometatarsal joints (Fig. 1) (Shively, 1982). These joints are relatively flat low-motion joints that allow mild rotation and translation of the distal tarsal region (Sisson, 1975), have an important shock absorbing function (Pool, 1996; Lanovaz et al., 2002) and are exposed primarily to compressive loading (Schamhardt et al., 1989). The conformation of the joints of the distal tarsal region means they are exposed to minimal shear forces compared to high motion metacarpo/metatarsophalangeal and carpal joints (Pool, 1996). Thus, OA lesion morphology and patterns are likely to vary between these different joint types.

Traditionally, light microscopy examination of paraffin- embedded specimens has been used as the reference standard to detect the earliest changes of OA in humans and animals (Laverty et al., 1991; McGibbon and Trahan, 2003; Batiste et al., 2004; Björnsdottir et al., 2004; Calvo et al., 2004; Bittersohl et al., 2009), and it has been used in studies of equine musculoskeletal disease to further investigate and verify changes seen with diagnostic imaging (Björnsdottir et al., 2004; Murray et al., 2006; Olive et al., 2009; Olive et al., 2010). Several studies have investigated the microscopic appearance of the joints in the distal tarsal region of horses using paraffin-embedded light microscopy. These studies include descriptions of lesion-free tarsometatarsal joints (Murray et al., 2009), descriptions of osteochondral lesions in the joints of the distal tarsal region of young horses and foals (Laverty et al., 1991; Watrous et al., 1991; Barneveld and van Weeren, 1999; Björnsdottir et al., 2004; Ley et al., 2013) and descriptions of osteochondral lesions in the centrodistal and tarsometatarsal joints of adult horses (Tranquille et al., 2011). A disadvantage of paraffin-embedding is that the technique requires decalcification of the tissues, which results in loss of mineralised tissue information.

A technique combining backscattered electron scanning electron microscopy (BSE SEM) and confocal scanning light microscopy (CSLM) using polymethyl methacrylate (PMMA)-embedded specimens results in extremely high resolution images of bone and cartilage with resolution down to $0.2 \mu \mathrm{m}$ (Boyde et al., 2005). With this method it is possible to see in unparalleled detail some of the commonly 
detected 'early OA' changes (Boyde et al., 2011). Recently, a BSE SEM method for scanning PMMA-embedded bone slab specimens that uses iodine-based staining methods prior to the BSE SEM scans has been developed and this results in microscopic morphological information for both the mineralised and non-mineralised tissues (Boyde, 2012b; Boyde, 2013). Unlike paraffin-embedding, PMMA-embedded BSE SEM and CSLM do not require any decalcification of the tissues so complete microscopic evaluation of the mineralised tissues of the joint is possible. This is particularly important in the region of the junction of the SCB and the articular calcified cartilage (ACC), and the 'tidemark' mineralising front (MF) at the junction of the hyaline articular cartilage (HAC) and the ACC.

The morphology of the articular aspect of the ACC MF can be studied en face using three-dimensional (3D) BSE SEM after removal of the HAC by maceration (Boyde and Firth, 2004; Boyde and Firth, 2008; Boyde et al., 2011). Using this method the topography of the articular aspect of the MF can be evaluated in regions up to several square centimetres in area and specific surface features can be evaluated with resolution down to $0.2 \mu \mathrm{m}$. Studies of the equine metacarpophalangeal joint using this method show changes that are considered to be related to osteochondral disease and OA (Boyde and Firth, 2008; Boyde et al., 2011), but no such studies have been published for the equine tarsus. To complement the en face MF information obtained with BSE SEM, microcomputed tomography $(\mu \mathrm{CT})$ can be useful to reveal the morphology of the mineralised tissues deep to the MF.

The only description of pathology in the joints of the equine distal tarsal region using BSE SEM is an example of hypermineralised cracks in the ACC in a review article (Boyde, 2003) and it is not known if the other osteochondral lesions detected in studies of the metacarpophalangeal joint (Boyde and Firth, 2004; Boyde and Firth, 2005; Doube et al., 2007; Boyde and Firth, 2008; Boyde et al., 2011) also occur in the distal tarsal joints.

The objective of this study was to investigate and characterise osteochondral lesion-types using a combination of microscopic methods that result in detailed images of the mineralised and non-mineralised tissues in the articular regions of the equine centrodistal, tarsometatarsal and the talocalcaneal-centroquartal joints (Fig. 1). The methods used included studies of macerated and PMMA-embedded material using BSE SEM, CSLM and $\mu$ CT techniques. Our hypothesis was that early morphological OA lesions occur primarily in the HAC and ACC.

\section{Materials and Methods}

The Icelandic horses that were used for this study were bred based on the radiological changes of OA detected in the parents' distal tarsal joints. Parent selection was used to breed a study group where the majority of the horses had a high risk for developing OA of the joints in the distal tarsal regions and the minority of horses had a low risk with the goal of providing joints showing the full spectrum of normal joints to those with more severe OA. Radiographic evidence of distal tarsal OA was present in both parents of
13 of the horses, in neither parent in five horses, and in the right distal tarsal region of one parent in four horses. The radiographic classification of the parent horses was made by consensus of three experienced equine radiologists (SB, $\mathrm{KH}$ and CJL) with the presence of either SCB sclerosis/ lysis, narrow or uneven joint space, marginal osteophyte, periarticular bone bridging or ankylosis considered to indicate $\mathrm{OA}$.

The material for this study consisted of left tarsal joints from twenty-two, untrained, Icelandic horses ( 9 females, 13 males) that had lived as a semi-free-range group in northern Iceland and were slaughtered at $30 \pm 1$ months of age. Immediately following slaughter the skin was removed and the tarsal joints were separated from the leg by sawing through the mid tibia and disarticulating the limb at the level of the metatarsophalangeal joint. Unopened joints were transported refrigerated for 24 to $50 \mathrm{~h}$ to a clinical diagnostic imaging facility where MRI and CT images of the joints were acquired to allow image guided sampling of osteochondral tissue for microscopy using a described method (Ley et al., 2013). Twelve to sixteen hours after completion of the MRI and CT studies the unopened distal tarsal joints were manually sectioned with a bandsaw (KT-400, Klaukkala, Finland) using parallel saw cuts to make 5-10 mm thick frontal plane slabs. The positioning and angulation of the saw cut was done according to a described method (Björnsdottir et al., 2004). Tarsal slabs were then immersed in $10 \%$ neutral buffered formalin or placed in plastic bags and frozen $\left(-20^{\circ} \mathrm{C}\right)$, with time between slaughter to tarsal slab fixation/freezing ranging from 45 to $69 \mathrm{~h}$. Tarsal slabs were transferred to $70 \%$ ethanol 5-7 d before sub-sampling and then into glycerine immediately after sampling.

\section{Osteochondral sampling guidance with CT and MRI} (Fig. 1)

Samples of the osteochondral tissue were taken from the centrodistal, tarsometatarsal and talocalcanealcentroquartal joints using a modification of a described CT and MRI guidance method (Ley et al., 2013). In brief, the tarsal slabs were imaged with clinical CT (Definition 64-slice, Siemens Medical Systems, Erlangen, Germany) to allow co-registration of tarsal slab images with the whole tarsus images. MRI and CT images of the entire distal tarsal regions were systematically examined and graded for abnormalities of the articular cartilage, SCB and the joint margins using a published method (Ley et al., 2013). For each joint the sites of the highest frequency and degree of abnormal features were identified by marking regions of interest on the whole tarsus CT images, and these regions of interest were identified and localised using image coregistration in the tarsal slab CT images. Up to four regions of interest were identified in each joint and joints with high frequencies of abnormal features had the most regions of interest marked. These regions of interest were ranked subjectively (CJL) within each joint according to the frequency and degree of abnormal features. The locations of the regions of interest were marked on $3 \mathrm{D}$ volumetric reconstructions of the bone slabs (OsiriX v 3.8, Geneva, Switzerland), which were used to guide specimen removal from the bone slabs. 
Table 2. Summary of the width and whether one or both articular surfaces were included in specimens from distal tarsal joints. A full width specimen extended from the lateral to the medial margin of the joint.

\begin{tabular}{|c|c|c|c|c|c|c|}
\hline Joint & $\begin{array}{l}\text { Full width of } \\
\text { both articular } \\
\text { surfaces }\end{array}$ & $\begin{array}{l}\text { Full width of } \\
\text { one articular } \\
\text { surface }\end{array}$ & $\begin{array}{l}\text { Partial width of } \\
\text { both articular } \\
\text { surfaces }\end{array}$ & $\begin{array}{l}\text { Partial width } \\
\text { of one articular } \\
\text { surface }\end{array}$ & $\begin{array}{l}\text { Full width of one and } \\
\text { partial width of the other } \\
\text { articular surface }\end{array}$ & $\begin{array}{l}\text { Total number of } \\
\text { joints sampled }\end{array}$ \\
\hline & \multicolumn{6}{|c|}{ PMMA-embedded specimens } \\
\hline TCCQJ & 1 & 4 & \begin{tabular}{l|l}
0 \\
\end{tabular} & \begin{tabular}{|c|}
2 \\
\end{tabular} & 0 & 7 \\
\hline CDJ & 19 & 0 & 2 & 1 & 0 & 22 \\
\hline \multirow[t]{2}{*}{ TMTJ } & 17 & 0 & 2 & 0 & 1 & 20 \\
\hline & \multicolumn{6}{|c|}{ Macerated specimens } \\
\hline TCCQJ & 3 & 1 & \begin{tabular}{l|l}
0 &
\end{tabular} & 1 & 0 & 5 \\
\hline CDJ & 6 & 1 & 1 & 1 & 0 & 9 \\
\hline TMTJ & 4 & 1 & 1 & 0 & 0 & 6 \\
\hline
\end{tabular}

$\mathrm{CDJ}=$ centrodistal joint; $\mathrm{TCCQJ}=$ talocalcaneal-centroquartal joint; $\mathrm{TMTJ}=$ tarsometatarsal joint; $\mathrm{PMMA}=$ polymethyl methacrylate.

\section{Osteochondral specimen removal from tarsal slabs} In order to select osteochondral specimens containing a high frequency and degree of OA lesions and examples of specific lesion-types, the number of specimens taken from each joint and the microscopic technique used for each specimen was chosen subjectively, based on the information in the MRI and CT images (AB, SE and CJL). The most severe lesion regions of interest in centrodistal joints (up to a maximum of four for each joint) were sampled using the CT and MRI guidance method (Ley et al., 2013) and these specimens often included adjacent tarsometatarsal and/or talocalcaneal-centroquartal joints. If possible, attempts were made to include multiple lesion regions of interest in one specimen. If no CT or MRI changes were detected in the centrodistal joint, then a specimen of the centrodistal joint was removed using a predetermined sampling method (Björnsdottir et al., 2004) without MRI/CT guidance. Lesion regions of interest in tarsometatarsal and talocalcaneal-centroquartal joints were then sampled and these specimens often included adjacent centrodistal joints or occasionally also tarsometatarsal and/ or talocalcaneal-centroquartal joints. All joints included in a specimen were subsequently evaluated with microscopy regardless of whether or not MRI or CT abnormalities had been detected.

Osteochondral specimens were subsampled using a diamond saw (Labcut, DR Bennett LTD, London, UK) with guidance from the MRI and CT images. For preparation of PMMA-embedded specimens, 1.5 to $4 \mathrm{~mm}$ thick osteochondral slabs were made. Osteochondral blocks 5 to $15 \mathrm{~mm}$ thick were taken for BSE SEM studies after maceration of non-mineralised material. Osteochondral slabs and blocks were marked with pencil to indicate the location of the guided sample site in the block and for block orientation, photographed and stored in glycerol.

\section{Processing and imaging of PMMA-embedded osteochondral specimens}

Osteochondral slab specimens were embedded in PMMA and prepared as plane parallel slabs (Boyde, 2012a). The examination surface was ground and polished using a graded series of silicon carbide papers and diamond abrasives down to one micron. The scan surface of each PMMA-embedded specimen was photographed for image navigation in the SEM.

PMMA-embedded specimens for BSE SEM were carbon-coated, and examined using a Zeiss DSM962 SEM (Zeiss UK Ltd, Welwyn Garden City, UK) at $20 \mathrm{kV}$ accelerating voltage with a 4 sector solid state BSE detector (KE Electronics, Toft, UK) as previously described (Boyde and Firth, 2005; Boyde and Firth, 2008). Later in the study a Zeiss EVO MA10 SEM (Carl Zeiss NTS Ltd, Cambridge, UK) was acquired, which enabled us to image uncoated PMMA-embedded specimens when operated at $50 \mathrm{~Pa}$ chamber pressure and $20 \mathrm{kV}$ for BSE imaging. Subsequently, procedures for staining non-mineralised cell and matrix components using iodine were developed by AB (Boyde, 2012b; Boyde, 2013). Blocks were re-polished to remove any carbon coating and stained with solutions of triiodide in potassium or ammonium iodide (triiodide ion) or stained dry with elemental iodine vapour, simply by placing the PMMA-embedded specimens in a sealed container with some iodine crystals.

Uncoated PMMA-embedded specimens examined with CSLM were cover-slipped with glycerol and examined using a Leica DMRBE with a SP2 confocal scan head and using 10/0.4 dry and 25/0.7 oil objectives (Leica Microsystems Ltd, Milton Keynes, UK). The $488 \mathrm{~nm}$ line from an argon ion laser was used for excitation, and autofluorescence emission was collected into green (band from 498-578 nm) and red (588-734 nm) channels.

The CSLM and SEM scans were done whilst making reference to the MRI and CT images and using 3D volumetric reconstructions of these images.

\section{Processing and imaging of macerated specimens}

Block specimens of joint surfaces were macerated using $2 \%$ Tergazyme (alkaline pronase enzyme detergent, Alconox Inc., New York, NY, USA) to remove HAC and other soft tissue, washed and air dried. If Tergazyme treatment failed to remove cartilage, tendon or ligament, the specimens were further treated by immersion in $5 \%$ 
hydrogen peroxide solution (six fold dilution of fresh stock concentrate) until clean. Once clean, the intended scan surface of all specimens was photographed. These specimens were either carbon-coated and examined using the Zeiss DSM962 SEM at $20 \mathrm{kV}$, or examined uncoated in the Zeiss EVO MA10 SEM operated at $50 \mathrm{~Pa}$ chamber pressure, and again at $20 \mathrm{kV}$ using BSE. Three-dimensional images were recorded by taking multiple images with $1^{\circ}$ or $2^{\circ}$ angle tilt difference and processed into movies to allow 3D to be appreciated through motion parallax; or by taking two images with a $6^{\circ}$ tilt angle difference giving a stereoscopic pair, and these were processed to give redcyan anaglyphs that could be viewed with colour-filter spectacles.

\section{$\mu C T$ imaging}

Further investigation of macerated and PMMA-embedded specimens was done with high resolution $\mu \mathrm{CT}$ (Scanco $\mu \mathrm{CT} 40$, Scanco Medical AG, Brüttisellen, Switzerland) when highly mineralised MF protrusions were seen or suspected. Macerated and PMMA-embedded specimens were cut and trimmed to a cross-sectional size of less than $7 \mathrm{~mm}$, permitting $6 \mu \mathrm{m} \mu \mathrm{CT}$ resolution.

\section{Examination of microscopy specimens}

Tissues from all horses $(n=22)$ were PMMA-embedded and included 7 talocalcaneal-centroquartal joints, 22 centrodistal joints and 20 tarsometatarsal joints (Table 2). In two centrodistal and three tarsometatarsal joints two separate specimens of the joint were taken and in three centrodistal and one tarsometatarsal joints there were three separate specimens taken. Thirty-nine joints were examined with only iodine-stained BSE SEM; four joints were examined first with CSLM then BSE SEM after carbon coating and after re-polishing were stained with iodine and re-examined with BSE SEM; six joints were examined carbon-coated with BSE SEM, re-polished, stained with iodine and then re-examined with BSE SEM. One PMMA-embedded specimen was examined with $\mu \mathrm{CT}$ after imaging with iodine-stained BSE SEM.

Tissues from 12 horses were macerated; this included five talocalcaneal-centroquartal joints, nine centrodistal joints and six tarsometatarsal joints (Table 2). BSE SEM was done of the joint surfaces and this was followed by $\mu \mathrm{CT}$ for two talocalcaneal-centroquartal joints and three centrodistal joints.

The BSE SEM and CSLM images were evaluated by three of the authors (AB, SE, and CJL) and the $\mu \mathrm{CT}$ images by two of the authors (AB and CJL). When multiple microscopic techniques were used, images were correlated to evaluate the range of lesions present. Lesion classifications (lesion-types) were proposed from the morphological changes observed in the specimens. Two of the authors (SE, CJL) systematically re-examined only the BSE SEM images and determined by consensus which lesion-types were present in each specimen. This information was used to record the frequency of osteochondral lesion-types in talocalcaneal-centroquartal, centrodistal and tarsometatarsal joints and to calculate associations and correlations between the lesion-types in the centrodistal joints.

\section{Statistical analysis}

The frequencies of lesion-types were recorded as percentages of joints examined according to joint (talocalcaneal-centroquartal, centrodistal or tarsometatarsal joint) and specimen type (PMMA-embedded or macerated). If multiple specimens were taken from an individual joint, then all of the lesion-types from all of the specimens from that joint contributed to the lesion-type frequency for that joint (pooled frequency of lesion-types per joint). A value of 0 (lesion-type not present) or 1 (lesion-type present) was given to each individual joint for each of the lesion-types in PMMA-embedded specimens or each of the lesion-types in macerated specimens in each joint examined.

Using the pooled lesion-type count data from the PMMA-embedded centrodistal joints Fisher's exact tests were performed to detect associations between the presence of osteochondral lesion-types. To control type-1 errors, the Benjamini and Hochberg false discovery rate procedure was applied to $p=0.05$ to determine significant $p$-values for individual tests of association (Benjamini and Hochberg, 1995). Phi coefficients were calculated from the same data to investigate the degree and direction of correlations between the osteochondral lesion-types. Phi coefficient values $>0.6$ were considered to indicate strong positive correlations, values 0.3 to 0.6 weak to moderate positive correlations, values between -0.3 to 0.3 little or no correlations, values -0.6 to -0.3 weak to moderate negative correlations and values $<-0.6$ strong negative correlations.

Statistical calculations for lesion-type associations and correlations were not possible for the talocalcanealcentroquartal and tarsometatarsal joints due to the low frequency of lesions in these joints.

All analyses were performed using the SAS statistical software program (SAS 9.2 software program, Cary, NC, USA).

\section{Results}

\section{Image variations of the microscopic techniques}

Images of joint regions obtained with BSE SEM and CSLM with no lesions detected showed joint morphology and the variations expected from the tissue preparation methods (Figs. 2 and 3). CSLM images of PMMA-embedded specimens resulted in excellent images of non-mineralised tissues but only limited information about the mineralised tissues (Figs. 2a, 3a). Images from BSE SEM of uncoated or carbon-coated PMMA-embedded specimens resulted in excellent images of the mineralised tissues but it was not possible to define morphological structures in the non-mineralised tissues (Figs. 2c, 3b). Maceration of joint surfaces and margins and subsequent scanning with BSE SEM produced en face images allowing evaluation of the MF topography of joint regions (Figs. 3d-e). PMMAembedded specimens with potassium or ammonium triiodide (Figs. 2d, 3c), or iodine vapour (Fig. 2b) staining for BSE SEM resulted in images showing the morphology of both the mineralised and non-mineralised osteochondral tissues. The chondrocyte lacunae were well defined and sometimes the chondrocyte nucleus and cytoplasm also stained. Variations in staining intensity and uniformity 

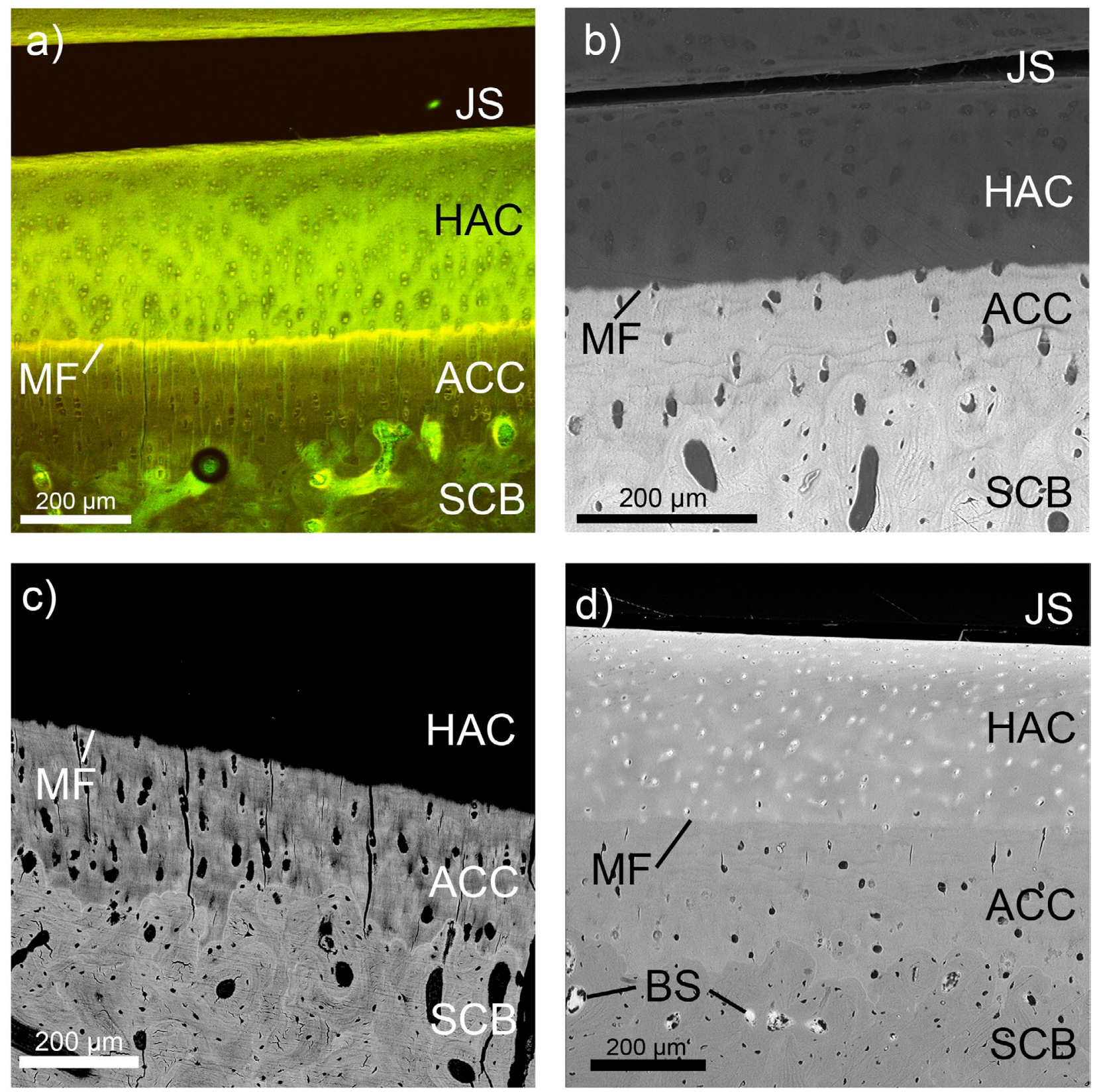

Fig. 2. Central regions of normal centrodistal joints from PMMA-embedded specimens. (a) CSLM shows the morphological details of the hyaline articular cartilage (HAC), but articular calcified cartilage (ACC) and subchondral bone (SCB) details are limited. (b) BSE SEM after iodine vapour staining shows stained HAC extra-cellular matrix. (c) Unstained BSE SEM where the HAC is invisible. (d) BSE SEM after triiodide solution staining shows stained HAC extra-cellular matrix and chondrocytes. The mineralising front (MF) is visible using all the microscopic techniques. (b-d) The morphology of the ACC and SCB seen in detail in all BSE SEM images, although the contrast resolution is reduced after iodine/triiodide staining. (d) Triiodide staining often resulted in high concentrations of triiodide stain in bone marrow spaces and vascular channels (BS). Joint space (JS).

were seen between specimens depending on the tissue penetration of the triiodide or iodine vapour stain, but this did not interfere with interpretation of tissue morphology (Figs. 2b, 2d). It was common for high concentrations of triiodide stain to be seen in bone marrow spaces due to the high affinity of iodine for the blood present in these spaces (Figs. 2d, 3c). Focal regions of accumulated dried triiodide stain were occasionally seen in imperfectly embedded areas in PMMA-embedded block surfaces.

\section{Normal articular osteochondral morphology}

Normal PMMA-embedded joint specimens showed smooth occasionally gently undulating uninterrupted MF, a wavy and bumpy cement line (ACC junction with SCB) with variable size and shaped projections of $\mathrm{SCB}$ extending into the deep surface of the ACC, and, in all techniques except unstained BSE SEM, a smooth occasionally undulating uninterrupted HAC articular surface (Figs. 2a-d, 3a-c). Chondrocytes within the HAC were evenly distributed 

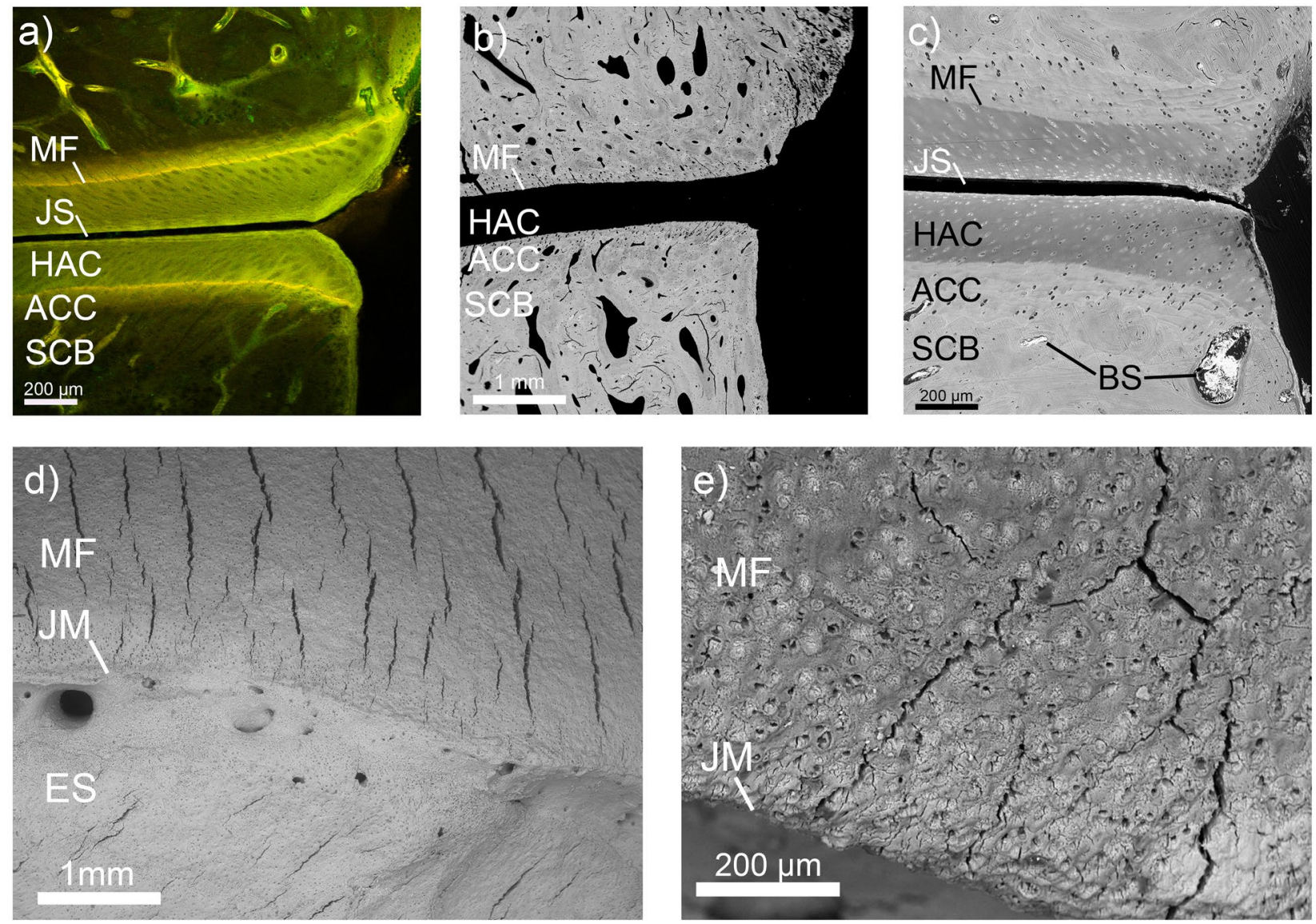

Fig. 3. Marginal regions of normal joints. (a-c) PMMA-embedded centrodistal joints, (d) macerated centrodistal joint, and (e) macerated tarsometatarsal joint specimens. (a) CSLM, (b) unstained BSE SEM, (c) triiodide stained BSE SEM, (d) low magnification unstained BSE SEM, and (e) high magnification unstained BSE SEM. The mineralising front (MF) is visible in cross-section in panels a-c and en face in panels d-e. Close to the joint margins the columns of chondrocytes in the HAC normally became moderately oblique to the articular surface (a) and (c). Articular calcified cartilage (ACC); bone marrow spaces containing a high concentration of triiodide stain (BS); external bone surface (ES); hyaline articular cartilage (HAC); joint margin (JM); joint space (JS); subchondral bone (SCB).

either singly or as doublets (Figs. 2b, 2d) or in the deep zone often in vertical columns (Fig. 2a), although in regions close to joint margins chondrocyte columns were often oblique to the articular surface (Figs. 3a, 3c). Normal joint margins had articular cartilage extending to the margin and on the non-articular surface of the margin a thin covering of fibrous joint capsule and periosteum (Figs. 3a, 3c). The density of chondrocyte lacunae in the ACC was less than in the HAC but spatial arrangement was similar (Figs. 2a-d, $3 \mathrm{~b}-\mathrm{c}$ ). Normal macerated joints had a smooth even MF with evenly spaced chondrocyte lacunae in the MF, rounded to angular joint margins and an ACC MF extending to the articular margin (Figs. 3d-e).

Cracking of the osteochondral tissue was common in both macerated and PMMA-embedded specimens (Figs. 2 and 3 ) and these cracks were considered, as has been seen in previous studies, as post-processing drying artefacts due to loss of firmly bound structural water on storage, accelerated by exposure to the SEM vacuum environment (Boyde, 2003; Boyde et al., 2011). Occasionally in PMMAembedded specimens there were remnants of saw debris in the intra-articular and peri-articular regions but this did not interfere with interpretation of tissue morphology.

\section{Lesion descriptions}

Descriptions of the lesion-types that were identified in specimens are provided in Tables 3 and 4. Examples of these lesion-types are shown in Figs. 4-9.

\section{Hyaline articular cartilage (HAC) lesion-types}

In the HAC the lesion-types chondrocyte loss, chondrocyte clusters and fibrillation were detected (Table 3, Figs 4a-d, 5a-d, 6b, 7a, 7c, 8d). Regions of chondrocyte loss (Figs. 4a-d, 5b-d, 7a, 7c, 8d) were always focal and commonly contained a coarse linear to wavy texture tissue, with striations usually parallel or mildly oblique to the articular surface due to replacement of hyaline cartilage with fibrocartilage-like tissue (Figs. 4a-b, 4d, 5c). During the initial image evaluation it was observed that small clusters of chondrocytes were common very close to the joint margin. To further investigate this, chondrocyte cluster lesion-types in the HAC were further subdivided into 
Table 3. Descriptions of lesion-types in PMMA-embedded specimens from joints of the distal tarsal region.

\begin{tabular}{|l|l|}
\hline Hyaline articular cartilage (HAC) lesion-types \\
\hline HAC chondrocyte loss & $\begin{array}{l}\text { HAC regions that contained empty chondrocyte lacunae, small often flattened faint silhouettes } \\
\text { of chondrocytes or hypo- or acellular regions (Figs. 4a-d, 5b-d, 7a, 7c, 8d) }\end{array}$ \\
\hline $\begin{array}{l}\text { HAC central chondrocyte } \\
\text { clusters }\end{array}$ & $\begin{array}{l}\text { Groups of chondrocytes within a chondron two or more cells wide located }>500 \mu \mathrm{m} \text { from the } \\
\text { joint margin (Figs. 4a-c, 5a-d, 6b, 8d) }\end{array}$ \\
\hline $\begin{array}{l}\text { HAC marginal chondrocyte } \\
\text { clusters }\end{array}$ & $\begin{array}{l}\text { Groups of chondrocytes within a chondron two or more cells wide located <500 } \mu \mathrm{m} \text { from the } \\
\text { joint margin (Fig. 5d, 9a) }\end{array}$ \\
\hline HAC fibrillation & Articular surface discontinuities including fissures, splits and erosions (Fig. 4c-d, 5d, 7a) \\
\hline Articular calcified cartilage (ACC) lesion-types \\
\hline ACC chondrocyte loss & Hypo- or acellular ACC regions (Figs. 4a, 4c-d) \\
\hline ACC chondrocyte clusters & Groups of chondrocytes within a chondron two or more cells wide (Figs. 4a-b, 5a-d) \\
\hline ACC arrest & $\begin{array}{l}\text { Areas of thin, moderate to high mineralisation density, hypo- or acellular ACC which when focal } \\
\text { caused a concave contour of the mineralising front (Figs. 4d, 5b, 5d, 6b, 7a, 8d ) }\end{array}$ \\
\hline ACC advancement & $\begin{array}{l}\text { Areas of thick ACC, which when focal caused a convex contour of the mineralising front. Poorly } \\
\text { defined and hazy mineralising front and superficial layer of low density ACC (Figs. 5b-d, 6b, } \\
7 \mathrm{c}, 8 \mathrm{~d})\end{array}$ \\
\hline Hypermineralised infill phase (HIP) lesion-types \\
\hline ACC HIP & $\begin{array}{l}\text { Focal linear or irregularly shaped HIP material within the ACC and occasionally extending into } \\
\text { the SCB (Figs. 7a-c) }\end{array}$ \\
\hline HIP protrusions & $\begin{array}{l}\text { Focal protrusions of HIP within the articular cartilage that extended from the ACC into the HAC } \\
\text { (Figs. 7c, 8d-e) }\end{array}$ \\
\hline Subchondral bone (SCB) lesion-types \\
\hline SCB resorption & Concave to circular regions of SCB resorption (Fig. 6b) \\
\hline Joint margin lesion-types & $\begin{array}{l}\text { The joint margin at the level of the articular cartilage was enlarged by mineralised fibrous joint } \\
\text { capsule and periosteum (Fig. 9a, 9c), mineralised fibrocartilage (Fig. 9b) or bone (Fig. 5b, 9d) }\end{array}$ \\
\hline Joint margin extensions & Patches or craters of bone resorption on non-articular aspects of the joint margin (Fig. 9c-d) \\
\hline Joint margin erosions &
\end{tabular}

Table 4. Descriptions of lesion types in macerated specimens from joints of the distal tarsal region.

\begin{tabular}{|l|l|}
\hline Articular calcified cartilage (ACC) lesion-types \\
\hline ACC chondrocyte clusters & Imprints in the mineralising front of chondrons two or more chondrocytes wide (Fig. 6a, 6c-d) \\
\hline ACC arrest & Grooves and furrows formed by concave mineralising front regions (Figs. 5e, 6a, 6d) \\
\hline ACC advancement & Ridges or mounds formed by convex mineralising front regions (Figs. 6a, 6c-d) \\
\hline Hypermineralised infill phase (HIP) lesion-types \\
\hline HIP protrusions & Focal protrusions of HIP through the mineralising front (Fig. 7d) \\
\hline Subchondral bone (SCB) lesion-types \\
\hline SCB resorption & Concave areas of resorption extending into the SCB (Figs. 6a, 9f-g) \\
\hline Joint margin lesion-types & \\
\hline Joint margin extensions & Extension or enlargement of the joint margin (Fig. 9f) \\
\hline Joint margin erosions & Patches or craters of bone resorption on non-articular aspects of the joint margin (Fig. 9e) \\
\hline
\end{tabular}

clusters located very close (within $\sim 500 \mu \mathrm{m}$ ) to the articular cartilage joint margin, called marginal chondrocyte clusters (Figs. 5d, 9a), and central chondrocyte clusters that were further than $\sim 500 \mu \mathrm{m}$ from the joint margin (Figs. 4a-c, $5 \mathrm{a}-\mathrm{d}, 6 \mathrm{~b}, 8 \mathrm{~d})$. Focal regions of HAC chondrocyte loss and often fibrillation in opposing articular surfaces with adjacent regions of HAC containing groups of sometimes very large central chondrocyte clusters were common (Figs. 4c-d, 5d).

\section{Articular calcified cartilage (ACC) lesion-types}

In the ACC the lesion-types chondrocyte loss, chondrocyte clusters, arrest and advancement were detected (Table 3 and 4, Figs. 4a-d, 5a-e, 6a-d, 7a, 7c-d, 8d). The classification ACC arrest was chosen to describe areas of the ACC where the morphological characteristics suggested that the usual advance of the ACC towards the articular surface had ceased (arrest) or markedly slowed, but resorption with replacement of the deep surface of the ACC with bone appeared to be continuing resulting in the ACC in affected regions being thin or absent (Figs. $4 d, 5 b, 5 d$, $6 \mathrm{~b}, 7 \mathrm{a}, 8 \mathrm{~d})$. In some regions of ACC arrest, complete loss of the ACC resulted in direct contact between the HAC and SCB (Figs. 4d, 6b). In ACC arrest regions the MF often contained small focal non-mineralised chondrocyte lacunae confluent with the HAC matrix that extended into the ACC and contained a single chondrocyte (Figs. 5b, 6b). Grooves and furrows formed by the concave MF in ACC arrest regions were clearly demonstrated in the en face SEM images of the macerated specimens (Figs. 5e, 6a, 

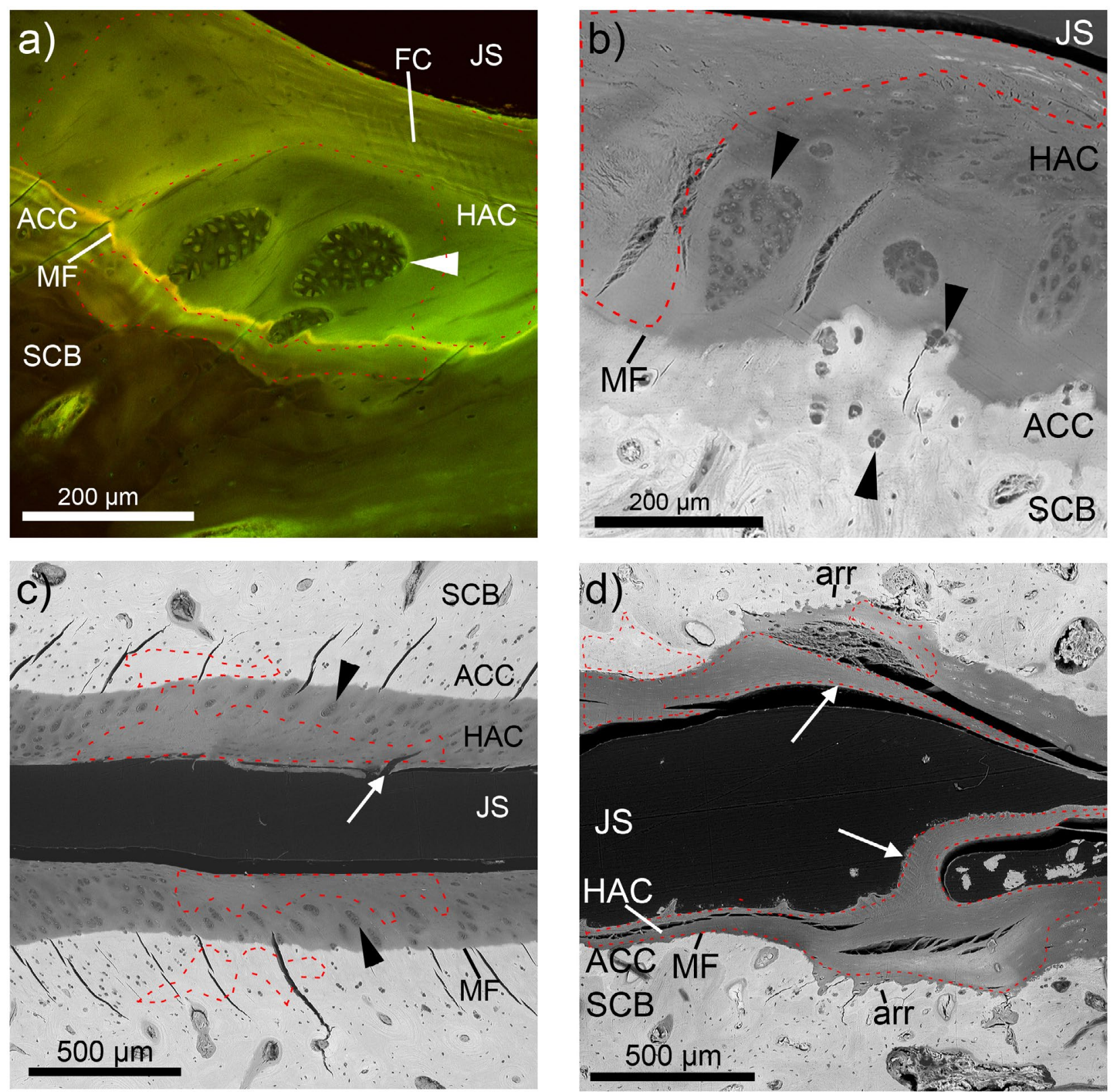

Fig. 4. Lesion-types in the hyaline articular cartilage (HAC) and articular calcified cartilage (ACC) of PMMAembedded centrodistal joint specimens. (a) CSLM, (b-c) BSE SEM stained with (b) triiodide, (c) iodine vapour, (d) triiodide. ACC arrest (arr); chondrocyte clusters (arrowheads); chondrocyte loss (regions within the red dashed lines); fibrocartilage-like tissue (FC); HAC fibrillation (arrows); joint space (JS), mineralising front (MF).

6d, 7d). Regions of ACC advancement typically formed a convex bulge of the MF (Figs. 5c-d, 6a-d, 8d) and often had a poorly defined hazy MF with a superficial layer of relatively low density mineralised cartilage, which was considered to be due to the active mineralisation taking place in this region of the ACC (Figs. 5b-c, 7c). Elongated peninsulas of mineralised cartilage in PMMA-embedded specimens were occasionally seen extending from the MF into the HAC to partially mineralised chondrocyte clusters in regions of ACC advancement, or, in other words, the MF advanced locally around chondrocyte clusters (Figs. $4 \mathrm{~b}, 5 \mathrm{~b}, 6 \mathrm{c})$. In and around regions of ACC advancement it was common to see chondrocyte clusters that were partially engulfed by the MF and thus within both the HAC and ACC (Figs. 4b, 5a-d). Regions of ACC arrest were often deep to HAC chondrocyte loss (Figs. 4d, 5b, 5d, 7a, 7c, $8 \mathrm{~d}$ ) and regions of ACC advancement were often deep to HAC central chondrocyte clusters (Figs. 5c-d, 6b, 8d).

Macerated and PMMA-embedded specimens taken from adjacent sites allowed the opportunity to correlate the appearance of the MF topography information from the macerated specimen with the cross-sectional tissue information from the PMMA-embedded specimen (Figs. $6 \mathrm{a}-\mathrm{b})$. Furrows and trenches in the MF corresponded to regions of ACC arrest and ridges corresponded to regions of ACC advancement. Often ACC arrest trenches followed the joint margin contour in macerated specimens (Fig. 5e) and ridges of ACC advancement were located either side of an ACC arrest with an advancement ridge between the joint margin and the arrest trench (Figs. 6a, 6d). In 

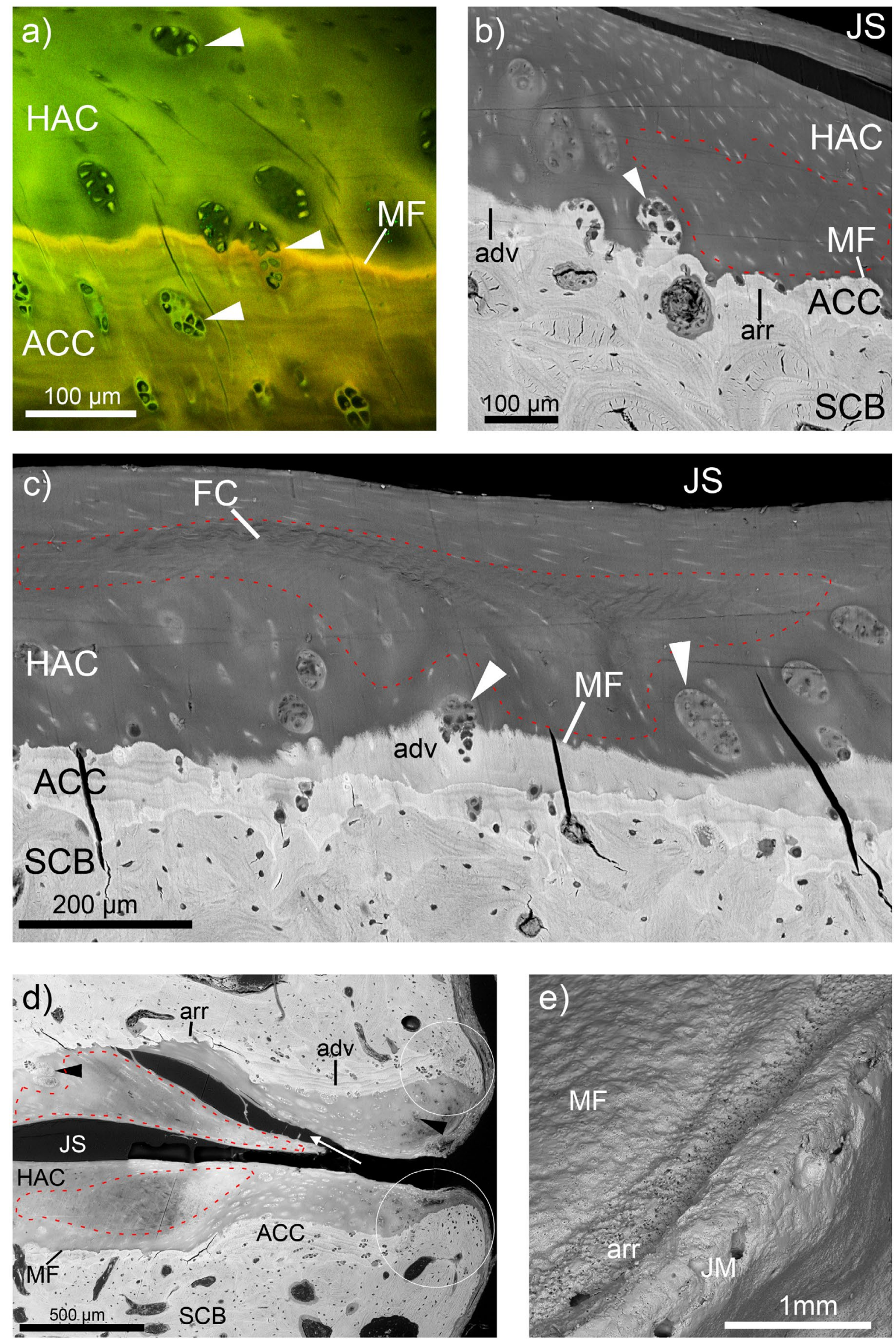

Fig. 5. Hyaline articular cartilage (HAC), articular calcified cartilage (ACC) and joint margin lesion-types. (a) CSLM and (b-e) BSE SEM images. (a-d) PMMA-embedded triiodide stained and (e) macerated en face centrodistal joint specimens. ACC advancement (adv); ACC arrest (arr); chondrocyte clusters (arrowheads); chondrocyte loss (regions within the red dashed lines); fibrocartilage-like tissue (FC); HAC fibrillation (arrow); joint margin (JM); joint margin extensions (within white circles); joint space (JS); mineralising front (MF); subchondral bone (SCB). 

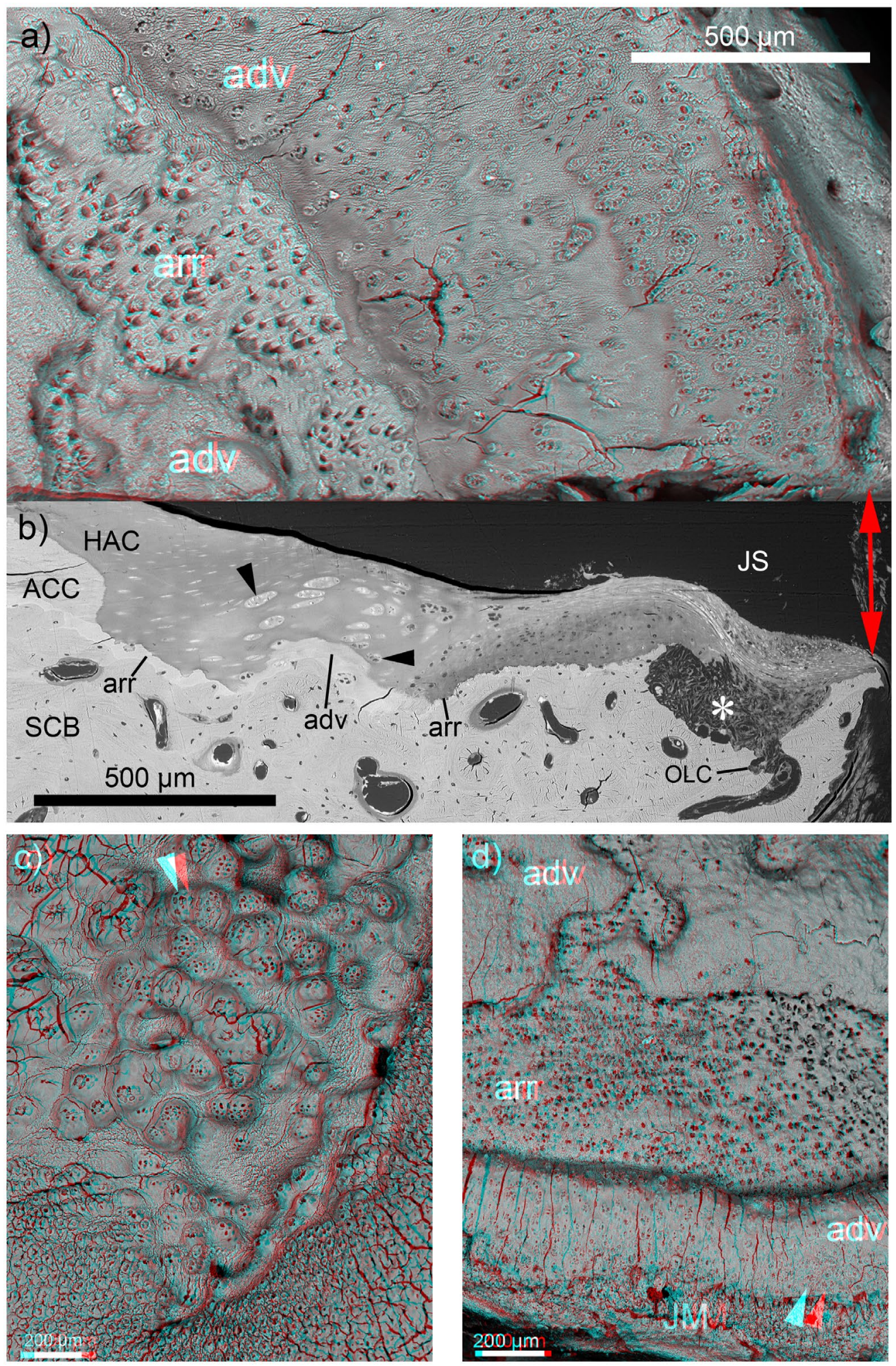

Fig. 6. Articular calcified cartilage (ACC), hyaline articular cartilage (HAC) and subchondral bone (SCB) lesion-types. BSE SEM images of (a,b,d) centrodistal joint and (c) talocalcaneal-centroquartal specimens. (a,c,d) Red/cyan anaglyph images of a macerated en face specimens. (a) Convex regions of ACC advancement (adv) and trenches of ACC arrest (arr). (b) PMMA-embedded triiodide-stained specimen that is adjacent the cut surface of the macerated specimen (separated by a bandsaw cut) shown in panel a. The adjacent joint margins of the two specimens are shown by the double headed red arrow. Note that due to loss of material in the bandsaw cut only a small part of the SCB resorption lesion (asterisk) seen in $b$ is visible in the macerated specimen in panel a (adjacent to the tip of the red arrow). An osteoclast-like cell (OLC) is seen within a resorption lacuna on the margins of the SCB resorption lesion. (c) Multiple large and partially mineralised chondrocyte clusters (arrowheads) protruding from the mineralising front. (d) ACC advancement lesions (adv) seen as convex bulges of the mineralising front separated by trenches of ACC arrest (arr). Impressions in the mineralising front resulting from chondrocyte clusters (arrowheads) that were partially engulfed by the mineralising front are seen on the ridge of ACC advancement (adv) that follows the joint margin (JM). Joint space (JS). 

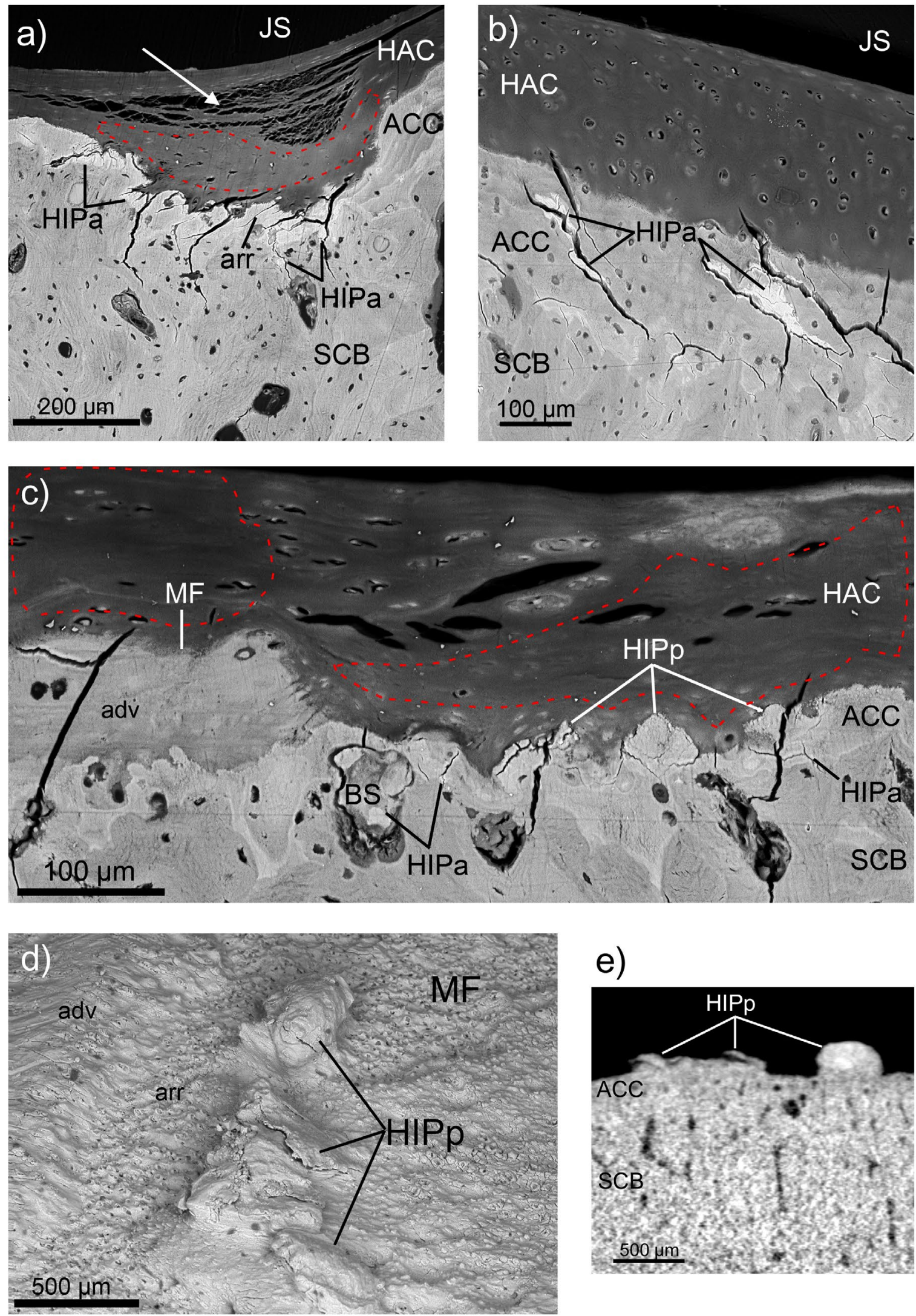

e)

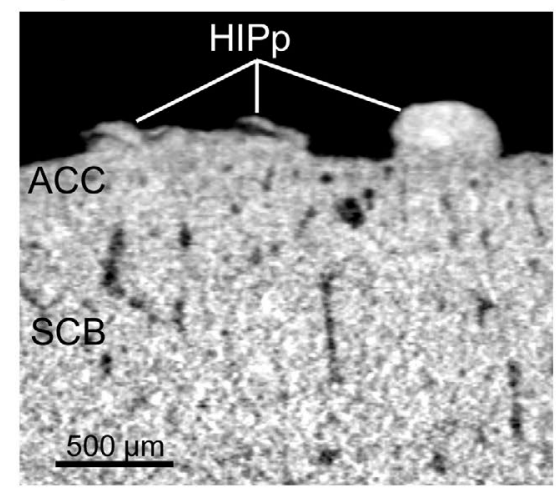

Fig. 7. Hypermineralised infill phase (HIP), hyaline articular cartilage (HAC) and articular calcified cartilage (ACC) lesion-types. (a-d) BSE SEM and (e) $\mu \mathrm{CT}$ images of (a) and (d-e) centrodistal joint and (b-c) tarsometatarsal joint specimens. (a-c) BSE SEM images of PMMA-embedded triiodide stained specimens. The HIP material is whiter than ACC and SCB. (d) En face BSE SEM, and (e) cross-sectional slice $\mu$ CT image of the same macerated specimen. The $\mu \mathrm{CT}$ image shows that the mineralising front (MF) protrusion detected with en face BSE SEM was hypermineralised and thus a HIP protrusion. ACC advancement (adv); ACC arrest (arr); bone marrow space (BS); chondrocyte loss (regions within the red dashed lines); HAC fibrillation (arrow); hypermineralised infill phase within ACC (HIPa); hypermineralised infill phase protrusion (HIPp). 

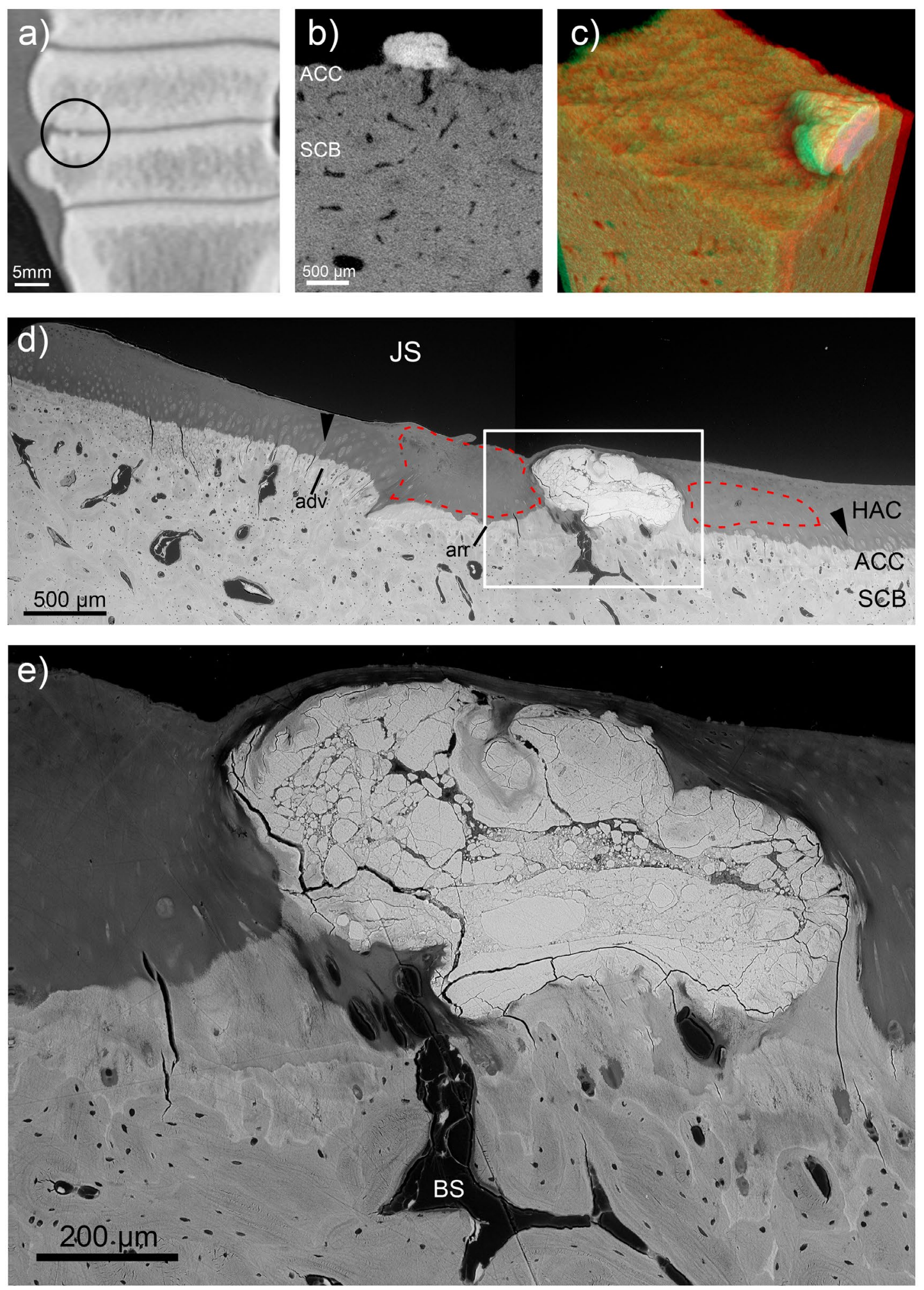

Fig. 8. Large hypermineralised infill phase (HIP) protrusion in a centrodistal joint. (a) Clinical CT image acquired before the tarsal joint was sectioned into slabs showing a focal hyperattenuating feature (centre of black circle) that was subsequently confirmed to be a HIP protrusion. (b) $\mu \mathrm{CT}$ image slice taken of the PMMA-embedded specimen that contained the hyperattenuating feature detected in the clinical CT. (c) 3D volumetric reconstruction red/cyan anaglyph to show both the cross-sectional structure and the shape and size of approximately half of this HIP protrusion. The adjacent mineralising front has an undulating topography caused by areas of articular calcified cartilage (ACC) arrest (arr) and ACC advancement (adv). (d) BSE SEM of a PMMA-embedded triiodide stained specimen that corresponds in location to the $\mu \mathrm{CT}$ images shown in panels b) and c) and demonstrating that this is a HIP protrusion that is confluent with the ACC and extends into the hyaline articular cartilage (HAC). (e) Higher magnification of the HIP protrusion shown within the white frame in panel $\mathrm{d}$. The HIP protrusion is composed of multiple mineralised fragments and no cells. A large bone marrow space (BS) extends from the SCB to the underside of the HIP protrusion. Chondrocyte clusters (arrowheads); chondrocyte loss (regions within the red dashed lines); joint space (JS). 

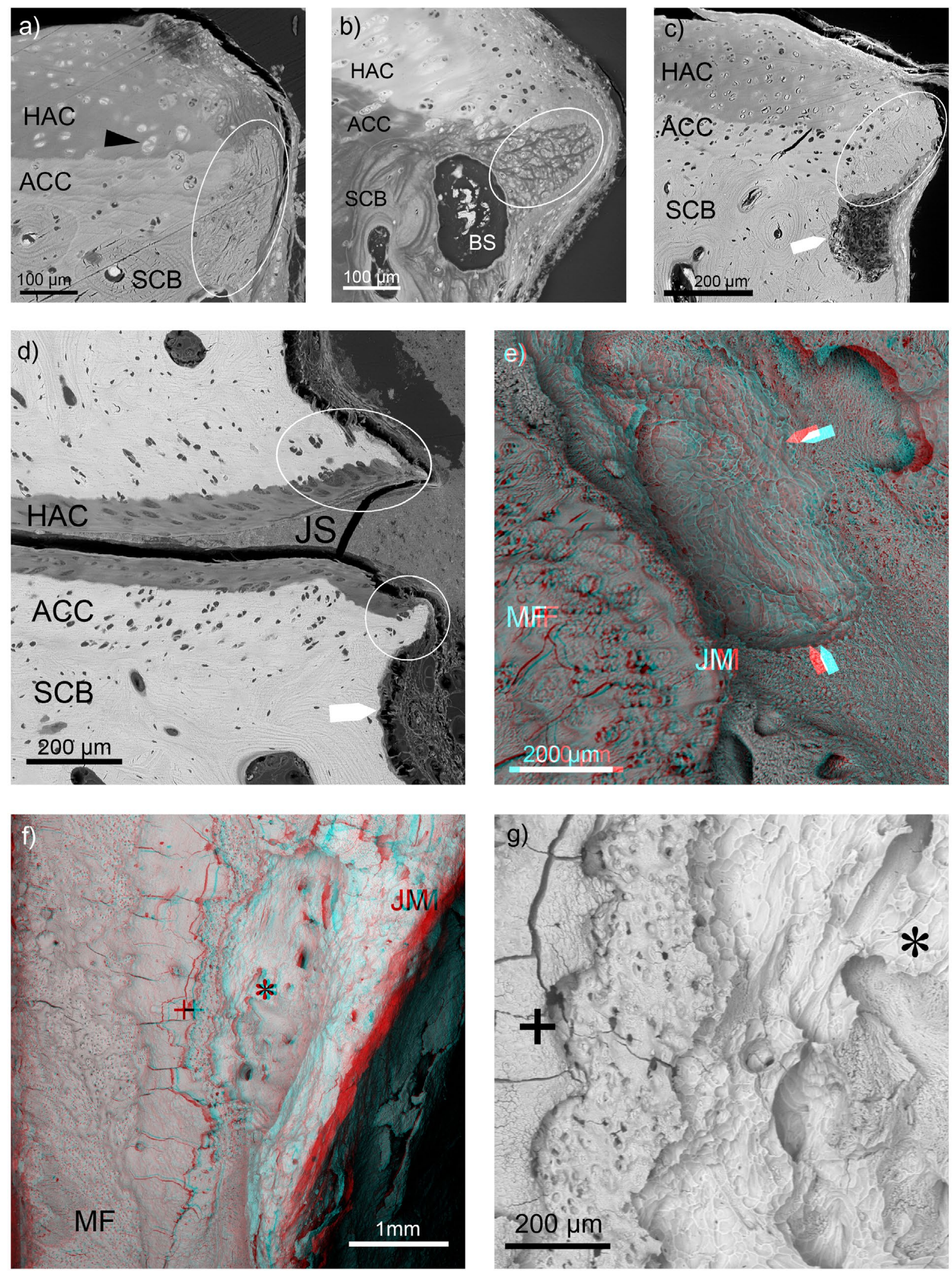

Fig. 9. Joint margin (JM) and subchondral bone (SCB) lesion-types. BSE SEM images of (a,c-g) centrodistal joint and (b) tarsometatarsal joint specimens. (a-d) PMMA-embedded triiodide-stained and (e-g) macerated specimens. Joint margin extensions (within white ellipse) from several joints include: (a,c,d) mineralisation of the periosteum/joint capsule; (b) mineralisation of fibrocartilage-like tissue with an adjacent large bone marrow space (BS) containing triiodide-stained blood. (c,d) Joint margin extensions and adjacent joint margin erosions (compact arrow) that result in an elongated spur shape of the joint margin. (e) A patch of joint margin erosion in a macerated specimen. (f) Red/cyan anaglyph image showing a region of joint margin extension with an adjacent SCB resorptive lesion (asterisk). (g) A magnified view of the edge of the SCB resorption lesion shown in $\mathrm{f}$ and the locations of the plus and the asterisk symbols are the same in both images. Articular calcified cartilage (ACC); hyaline articular cartilage (HAC). 


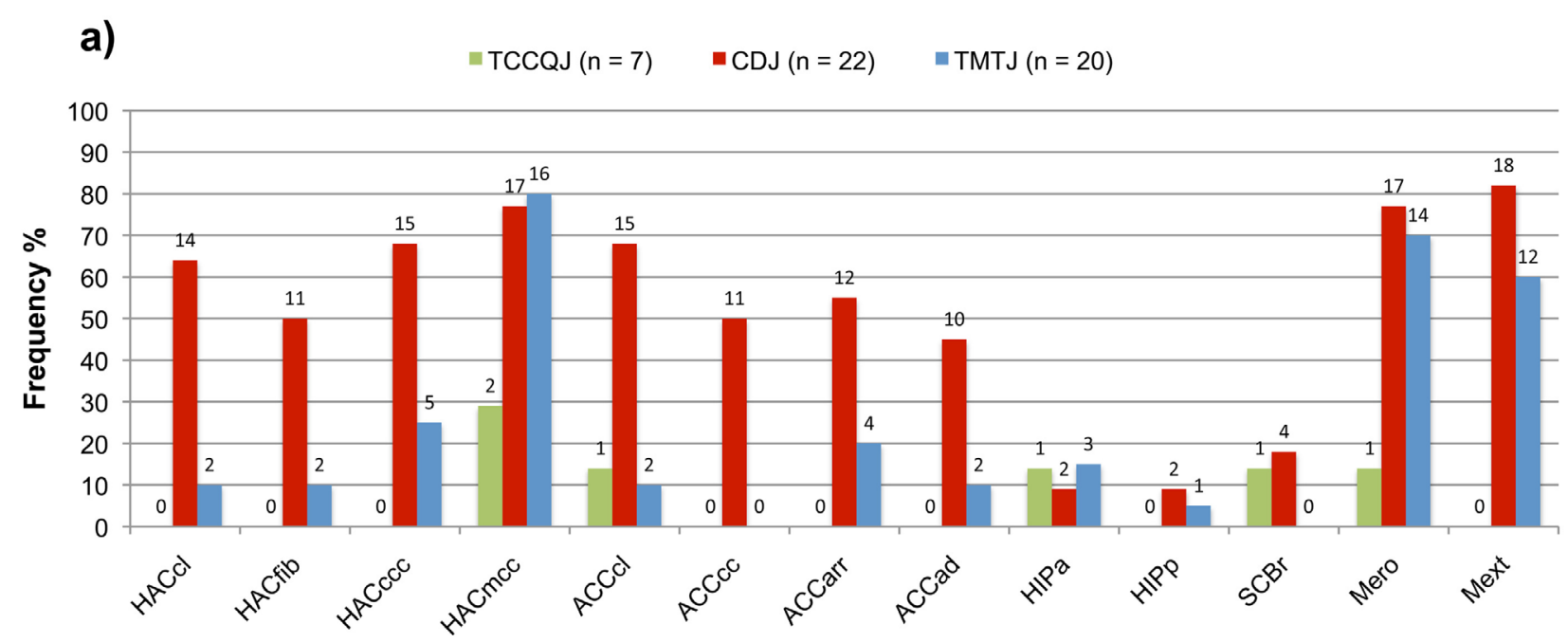

PMMAe specimen lesion type

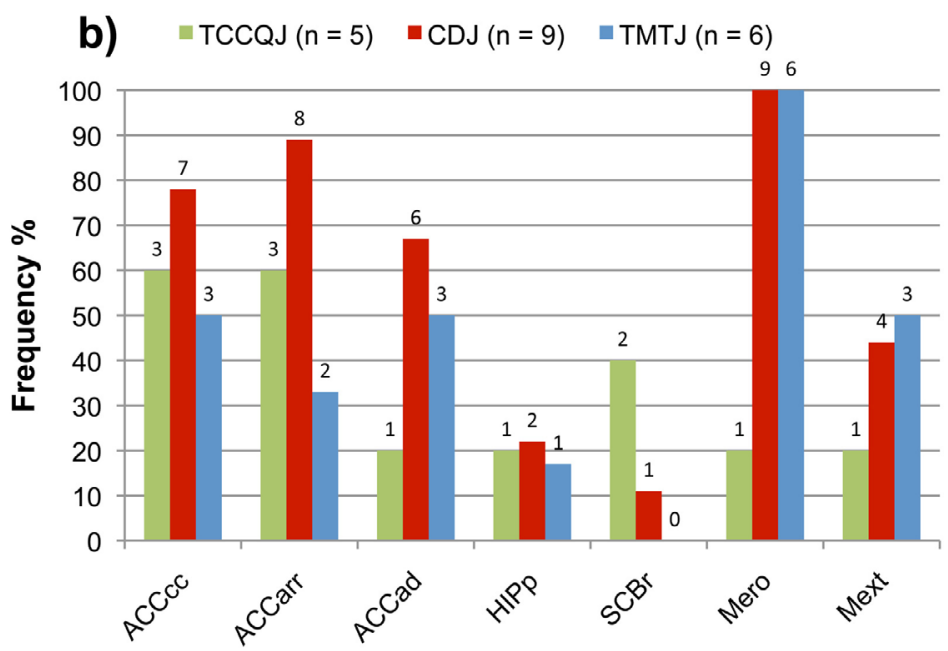

Macerated specimen lesion type

Fig. 10. Graphs showing frequency of osteochondral lesion-types detected in the talocalcaneal-centroquartal, centrodistal and tarsometatarsal joints as a percentage of total number of joints examined. Total number of joints examined is shown in the graph legend and number of joints detected with each lesion-type is shown at the top of each column. (a) Lesion-type frequency for PMMA-embedded specimens, and (b) lesion-type frequency for the macerated specimens. ACC $=$ Articular calcified cartilage; ACCad = ACC advancement $;$ ACCarr $=$ ACC arrest; $\mathrm{ACCcc}=\mathrm{ACC}$ chondrocyte cluster $; \mathrm{ACCcl}=\mathrm{ACC}$ chondrocyte loss; $\mathrm{CDJ}=$ centrodistal joint; HAC $=\mathrm{Hyaline}$ articular cartilage; $\mathrm{HACccc}=\mathrm{HAC}$ central chondrocyte cluster $; \mathrm{HACcl}=\mathrm{HAC}$ chondrocyte loss; HACfib $=\mathrm{HAC}$ fibrillation; HACmcc = HAC marginal chondrocyte cluster; HIPa = Hypermineralised infill phase within ACC; HIPp $=$ Hypermineralised infill phase protrusions; Mero $=$ Joint margin erosion; Mext $=$ Joint margin extension; $\mathrm{SCBr}=$ Subchondral bone resorption; TCCQJ $=$ talocalcaneal-centroquartal joint; TMTJ $=$ tarsometatarsal joint.

macerated specimens, chondrocyte clusters in regions of ACC advancement could be seen within the MF either as focal small imprints representing the lacunae that had been occupied by the chondrocytes (Figs. 6a, 6d) or partially mineralised chondrocyte clusters that protruded from the MF (Fig. 6c), whereas in other regions of ACC advancement the MF showed a broad smooth convex shape (Fig. 6d).

Hypermineralised infill phase (HIP) lesion-types Regions of HIP were seen within the articular cartilage and occasionally the SCB. This change was divided into the two lesion-types for PMMA-embedded specimens (Table 3): focal HIP within the ACC and occasionally extending into the SCB and bone marrow spaces (Figs. 7a-c) and HIP protrusions from the ACC into the HAC (Figs. 7c-e, $8 \mathrm{a}-\mathrm{e})$. There was always an increase in crack artefacts in the HIP material in the PMMA-embedded specimens resulting from specimen super-drying in the SEM (Figs. 7a-c, 8d-e). In macerated specimens, focal irregularly shaped protrusions from the MF were determined with subsequent $\mu \mathrm{CT}$ examinations to be hypermineralised and thus HIP protrusions from the MF (Figs. 7d-e). The $\mu \mathrm{CT}$ images of the macerated specimens did not show evidence 
of extension of the HIP protrusions from the articular cartilage into the SCB and no visible alterations in the structural arrangement of the SCB (Fig. 7e). This may have been because the HIP protrusions were entirely within the articular cartilage or that the resolution of the $\mu \mathrm{CT}$ was not high enough to detect very small SCB changes.

Information from the clinical $\mathrm{CT}$ images used in the image guided sampling of one centrodistal joint suggested that highly mineralised material was likely to be present within one of the PMMA-embedded specimens (Fig. 8a), even though evidence of this material was not seen in the initial BSE SEM images of the triiodide-stained block surface. Examination of this PMMA-embedded specimen with $\mu \mathrm{CT}$ (Figs. 8b-c) allowed identification of the location of the HIP protrusion and this region was scanned with BSE SEM, sequentially re-surfacing, re-polishing, repeating the triiodide staining and SEM study of the block surface until the level of the change was reached (Figs. 8d-e). The HIP protrusion projected above the level of the MF, was confluent with the ACC, contained within the HAC, displaced the surrounding $\mathrm{HAC}$ and did not cross through the HAC articular surface. Within an approximately $500 \mu \mathrm{m}$ sized region of the HIP protrusion there was HAC and ACC chondrocyte loss and ACC arrest, and further from the HIP protrusion there were chondrocyte clusters, areas of hypercellular HAC and ACC and evidence of advancement of the ACC (Figs. 8d-e).

\section{Subchondral bone (SCB) lesion-types}

$\mathrm{SCB}$ resorption lesions were filled with a mixture of fibrous tissue, blood vessels and occasional multinucleated osteoclast-like cells within small resorption lacunae at the bone interface (Fig. 6b). Blood vessels were seen entering the deep aspect of SCB resorption lesions (Fig. $6 \mathrm{~b})$. Articular aspect erosions adjacent the joint margin but that extended into the SCB were considered to be SCB resorption lesions (Figs. 6a, 9f-g).

An attempt was made to identify abnormalities in osteon numbers, size and appearance, but the active bone turnover in these young horses and the regular regional variation of the SCB plate thickness and bone marrow space numbers and sizes within the joints resulted in interpretation uncertainty. Thus lesions of abnormal bone turnover and SCB plate thickness were not defined.

\section{Joint margin lesion-types}

Mineralised extensions and erosions were identified as lesion-types of the joint margins (Figs 5d, 9a-e, 9f) and it was common to see joint margin extension and erosion together (Figs. 9c-d). Sharpey's fibres were often seen within mineralised joint margin extensions (Figs 9a, 9c).

\section{Lesion-type frequency in PMMA-embedded specimens}

Lesions were detected in all centrodistal joints that had PMMA-embedded specimens taken $(n=22)$ and all 13 lesion-types were detected (Fig. 10a). Lesion-type frequencies in the centrodistal joint were moderate to high in the HAC, variable in the $\mathrm{ACC}$, low in the SCB, high on the joint margins and low for HIP lesions.
Lesions were detected in all tarsometatarsal joints that had PMMA-embedded specimens taken $(n=20)$ and 11 of 13 lesion-types were detected (Fig. 10a). Lesion-type frequencies in the HAC of the tarsometatarsal joints were low except for marginal chondrocyte clusters, which had a high frequency similar to that seen in the centrodistal joint. Lesion-types of the tarsometatarsal joint margins had a moderate to high frequency with joint margin erosions having a similar frequency to that seen in the centrodistal joints.

Lesion-type frequencies in the talocalcanealcentroquartal joints were low (Fig. 10a). Only five of 13 lesion-types were identified and lesions were detected in only three of seven joints. In one talocalcanealcentroquartal joint the only lesion-type detected was marginal chondrocyte clusters in the HAC, and in another the only lesion-type detected was HIP within the ACC.

\section{Lesion-type frequency in macerated specimens}

Lesions were detected in all centrodistal joints that had macerated specimens taken $(n=9)$ and all seven lesiontypes were detected (Fig. 10b). Lesion-types of the ACC in the centrodistal joints had a moderate to high frequency. Margin erosions were detected in all the centrodistal joints examined and margin extensions were detected in four joints $(44 \%)$.

Lesions were detected in all tarsometatarsal joints that had macerated specimens examined $(n=6)$ and the only lesion-type not detected was SCB resorption (Fig. 10b). The tarsometatarsal joints had a mildly lower frequency of ACC lesion-types compared to the centrodistal joints. The frequency of joint margin lesion-types was almost identical to that of the centrodistal joints.

Four of the five talocalcaneal-centroquartal joints that had macerated specimens taken had at least one lesion detected and all seven lesion-types were detected in these joints (Fig. 10b). Similar to the frequency pattern in PMMA-embedded specimens, marginal lesions of the talocalcaneal-centroquartal joint in macerated specimens had a low frequency (Fig. 10b).

\section{Associations and correlations between lesion-types in PMMA-embedded centrodistal joint specimens}

Seventy-eight Fisher's exact tests of association were done and calculations using the Benjamini and Hochberg false discovery rate indicated $p$-values $\leq 0.0053$ were significant. Centrodistal joint lesion-types with moderate to high frequency that had significant associations and strong positive correlations were: HAC chondrocyte loss, HAC central chondrocyte clusters, HAC fibrillation, ACC arrest and ACC advancement; and ACC chondrocyte loss, HAC chondrocyte loss and HAC central chondrocyte clusters (Table 5). Lesion-types with high frequency that had no significant associations with other joint lesion-types were HAC marginal chondrocyte clusters and lesion-types of the joint margins (Table 5).

No significant associations were found for the low frequency lesion-types HIP protrusions, HIP within ACC and SCB resorption, although several Phi correlation values for these lesion-types suggested weak to moderate 
Table 5. Results of tests for correlation $(P h i)$ and association $(p)$ between lesion types detected in the PMMA-embedded centrodistal joints $(n=22)$.

\begin{tabular}{|c|c|c|c|c|c|c|c|c|c|c|c|c|c|}
\hline & & HACcl & HACfib & НАСсcс & HACmcc & ACCcl & ACCec & ACCarr & ACCad & HIPa & HIPp & $\mathrm{SCBr}$ & Mext \\
\hline Mero & $\begin{aligned} P h i & = \\
p & =\end{aligned}$ & $\begin{array}{l}0.27 \\
0.31\end{array}$ & $\begin{array}{c}0.11 \\
1\end{array}$ & $\begin{array}{c}0.1 \\
1 \\
\end{array}$ & \begin{tabular}{|c|}
-0.29 \\
0.29 \\
\end{tabular} & $\begin{array}{c}0.1 \\
1 \\
\end{array}$ & $\begin{array}{l}0.33 \\
0.31 \\
\end{array}$ & $\begin{array}{l}0.38 \\
0.14\end{array}$ & $\begin{array}{l}0.28 \\
0.32 \\
\end{array}$ & $\begin{array}{l}-0.21 \\
0.41 \\
\end{array}$ & $\begin{array}{c}0.17 \\
1\end{array}$ & $\begin{array}{l}0.26 \\
0.54\end{array}$ & $\begin{array}{c}0.03 \\
1\end{array}$ \\
\hline Mext & $\begin{aligned} P h i & = \\
p & =\end{aligned}$ & $\begin{array}{c}0.13 \\
0.6\end{array}$ & $\begin{array}{l}0.24 \\
0.59\end{array}$ & $\begin{array}{l}0.18 \\
0.56\end{array}$ & $\begin{array}{l}0.31 \\
0.21\end{array}$ & $\begin{array}{l}0.18 \\
0.56\end{array}$ & $\begin{array}{c}0.00 \\
1\end{array}$ & $\begin{array}{c}0.04 \\
1\end{array}$ & $\begin{array}{l}0.19 \\
0.59\end{array}$ & $\begin{array}{c}-0.26 \\
0.34\end{array}$ & $\begin{array}{c}-0.26 \\
0.34\end{array}$ & $\begin{array}{l}0.22 \\
0.55\end{array}$ & \\
\hline SCBr & $\begin{aligned} P h i & = \\
p & =\end{aligned}$ & $\begin{array}{l}0.36 \\
0.25\end{array}$ & $\begin{array}{l}0.47 \\
0.09\end{array}$ & $\begin{array}{l}0.32 \\
0.26\end{array}$ & $\begin{array}{l}0.26 \\
0.54\end{array}$ & $\begin{array}{c}0.07 \\
1\end{array}$ & $\begin{array}{l}0.47 \\
0.09\end{array}$ & $\begin{array}{c}0.43 \\
0.1\end{array}$ & $\begin{array}{l}0.52 \\
0.03\end{array}$ & $\begin{array}{c}0.15 \\
1\end{array}$ & $\begin{array}{l}0.44 \\
0.19\end{array}$ & & \\
\hline HIPp & $\begin{aligned} P h i & = \\
p & =\end{aligned}$ & $\begin{array}{l}0.24 \\
0.52\end{array}$ & $\begin{array}{l}0.32 \\
0.48\end{array}$ & $\begin{array}{c}0.22 \\
1\end{array}$ & $\begin{array}{c}0.17 \\
1\end{array}$ & $\begin{array}{c}0.22 \\
1\end{array}$ & $\begin{array}{l}0.32 \\
0.48\end{array}$ & $\begin{array}{l}0.29 \\
0.48\end{array}$ & $\begin{array}{l}0.35 \\
0.19\end{array}$ & $\begin{array}{l}0.45 \\
0.18\end{array}$ & & & \\
\hline HIPa & $\begin{aligned} P h i & = \\
p & =\end{aligned}$ & $\begin{array}{l}0.24 \\
0.52\end{array}$ & $\begin{array}{l}0.32 \\
0.48\end{array}$ & $\begin{array}{c}0.22 \\
1\end{array}$ & $\begin{array}{c}0.17 \\
1\end{array}$ & $\begin{array}{c}0.22 \\
1\end{array}$ & $\begin{array}{l}0 \\
1\end{array}$ & $\begin{array}{l}0.29 \\
0.48\end{array}$ & $\begin{array}{l}0.35 \\
0.19\end{array}$ & & & & \\
\hline ACCad & $\begin{aligned} P h i & = \\
p & =\end{aligned}$ & $\begin{array}{c}0.69 \\
0.0017^{\mathrm{a}}\end{array}$ & $\begin{array}{c}0.73 \\
0.0019^{\mathrm{a}}\end{array}$ & $\begin{array}{c}0.62 \\
0.0053^{\mathrm{a}}\end{array}$ & $\begin{array}{l}0.28 \\
0.32\end{array}$ & $\begin{array}{l}0.43 \\
0.07\end{array}$ & $\begin{array}{l}0.55 \\
0.03\end{array}$ & $\begin{array}{c}0.83 \\
0.0001^{\mathrm{a}}\end{array}$ & & & & & \\
\hline ACCarr & $\begin{aligned} P h i & = \\
p & =\end{aligned}$ & $\begin{array}{c}0.83 \\
0.0001^{\text {a }}\end{array}$ & $\begin{array}{c}0.73 \\
0.0019^{\mathrm{a}}\end{array}$ & $\begin{array}{c}0.75 \\
0.0007^{\mathrm{a}}\end{array}$ & $\begin{array}{l}0.16 \\
0.62\end{array}$ & $\begin{array}{l}0.55 \\
0.02\end{array}$ & $\begin{array}{l}0.55 \\
0.03\end{array}$ & & & & & & \\
\hline ACCec & $\begin{aligned} P h i & = \\
p & =\end{aligned}$ & $\begin{array}{l}0.57 \\
0.02\end{array}$ & $\begin{array}{l}0.45 \\
0.09\end{array}$ & $\begin{array}{l}0.49 \\
0.06\end{array}$ & $\begin{array}{l}0.33 \\
0.31\end{array}$ & $\begin{array}{c}0.2 \\
0.64\end{array}$ & & & & & & & \\
\hline ACCcl & $\begin{aligned} P h i & = \\
p & =\end{aligned}$ & $\begin{array}{c}0.70 \\
0.0023^{\mathrm{a}}\end{array}$ & $\begin{array}{l}0.49 \\
0.06\end{array}$ & $\begin{array}{c}0.79 \\
0.0006^{\mathrm{a}}\end{array}$ & $\begin{array}{l}0.33 \\
0.27\end{array}$ & & & & & & & & \\
\hline HACmcc & $\begin{aligned} P h i & = \\
p & =\end{aligned}$ & $\begin{array}{l}0.27 \\
0.31\end{array}$ & $\begin{array}{l}0.54 \\
0.04\end{array}$ & $\begin{array}{l}0.33 \\
0.27\end{array}$ & & & & & & & & & \\
\hline НАСсcс & $\begin{aligned} P h i & = \\
p & =\end{aligned}$ & $\begin{array}{c}0.90 \\
<0.0001^{\mathrm{a}}\end{array}$ & $\begin{array}{c}0.68 \\
0.0039^{\mathrm{a}}\end{array}$ & & & & & & & & & & \\
\hline HACfib & $\begin{aligned} P h i & = \\
p & =\end{aligned}$ & \begin{tabular}{|c|}
0.76 \\
$0.001^{\mathrm{a}}$
\end{tabular} & & & & & & & & & & & \\
\hline
\end{tabular}

Phi coefficient values $>0.6$ were considered strong positive correlation, values 0.3 to 0.6 weak to moderate positive correlation, values between -0.3 to 0.3 little or no correlation, values -0.6 to 0.3 weak to moderate negative correlation, and values $<-0.6$ strong negative correlation. Tests for association were made with Fisher's exact test. The level of significance was adjusted using the Benjamini and Hochberg false discovery rate applied to $p=0.05$, which resulted in a level of significance of $p \leq 0.0053$. Significant associations are marked with a superscript 'a'. ACC $=$ Articular calcified cartilage; $\mathrm{ACCad}=\mathrm{ACC}$ advancement; $\mathrm{ACCarr}=\mathrm{ACC}$ arrest; $\mathrm{ACCcc}=\mathrm{ACC}$ chondrocyte cluster; $\mathrm{ACCcl}$ $=\mathrm{ACC}$ chondrocyte loss; $\mathrm{HAC}=$ Hyaline articular cartilage; $\mathrm{HACccc}=\mathrm{HAC}$ central chondrocyte cluster; $\mathrm{HACcl}=$ HAC chondrocyte loss; HACfib = HAC fibrillation; HACmcc $=$ HAC marginal chondrocyte cluster; HIPa $=$ ACC hypermineralised infill phase; HIPp = Hypermineralised infill phase protrusions; Mero = Marginal erosion; Mext = Marginal extension; $\mathrm{SCBr}=$ Subchondral bone resorption.

correlations (Table 5). Despite the lack of statistical association in the centrodistal joint, when the images from all cases of HIP protrusions $(n=7)$ and SCB resorption $(n=7)$ were evaluated from all joints and all examination techniques there were always multiple lesion-types in the adjacent ACC and HAC in PMMA-embedded specimens (Figs. 6b, 7c, 8d) and in the adjacent MF in macerated specimens (Figs. 6a, 7d). However, this was not the case for HIP within ACC $(n=6)$, which were seen both in specimens with other HAC and ACC lesion-types (Fig. 7a) and in specimens without any other lesions (Fig. 7b).

\section{Discussion}

This study provides the first comprehensive microscopic investigation that evaluates both the mineralised and nonmineralised articular tissues of the joints in the distal tarsal region of the horse. We identified a range of osteochondral lesion-types in the talocalcaneal-centroquartal, centrodistal and tarsometatarsal joints, localised the tissue location of the lesion-types and investigated associations and correlations between lesion-types in centrodistal joints.

Significant associations were found between HAC chondrocyte loss, HAC central chondrocyte clusters and HAC fibrillation, suggesting that these morphological changes are occurring simultaneously and thus may have the same initiating cause (Table 5). These lesion-types correspond to those that have been reported in studies of OA of the distal tarsal joints that utilised light microscopy examination of paraffin-embedded specimens (Laverty et al., 1991; Barneveld and van Weeren, 1999; Björnsdottir et al., 2004; Tranquille et al., 2011; Ley et al., 2013). Due to these associations and patterns, we strongly suspect that the regions of HAC chondrocyte loss in our specimens were the result of chondrocyte necrosis. Cartilage destruction is one of the hallmark signs of OA (Mankin and Lippiello, 1970; Pritzker et al., 2006; Heinegard and Saxne, 2011) and the combination chondrocyte loss, fibrillation and chondrocyte clusters in HAC fulfils the criteria to be considered morphological OA (Mankin and Lippiello, 1970; Pritzker et al., 2006; McIlwraith et al., 2010). The 
focal distribution of the HAC lesions, which were often on opposing articular surfaces, is consistent with these being early OA changes (Dieppe and Kirwan, 1994; Squires et al., 2003; Veje et al., 2003; Pritzker et al., 2006). Thus we consider that the joints that had the combination of these HAC lesion-types to represent a group of joints with early morphological OA, and expect that if lesions of other joint tissues are part of OA development then those lesions would be associated and correlated with the HAC lesions.

The general patterns of lesion frequency (Figs. 10a-b) that we detected in the distal tarsal joints of these young Icelandic horses agree with studies of distal tarsal OA in adult horses further supporting that the changes we have detected are related to the early stages of OA. Radiographic studies of adult horses consistently show that the frequency and severity of OA lesions is highest in centrodistal joints compared to tarsometatarsal joints (Eksell et al., 1999; Labens et al., 2007; Byam-Cook and Singer, 2009; Fairburn et al., 2010) and that the talocalcaneal-centroquartal joints have the lowest incidence of OA lesions (Eksell et al., 1999; Labens et al., 2007). Other studies also investigate the frequencies of marginal changes compared to other joint lesions in adult horses (Labens et al., 2007; Fairburn et al., 2010) and, in agreement with our results, these studies report the frequencies of marginal osteophytes in the centrodistal and tarsometatarsal joints to be high and similar, but that lesions of the joint space region are more numerous in the centrodistal joint compared to the tarsometatarsal joint. A radiographic and histological study also reports similar lesion frequency patterns in horses ranging from 6 months to 13 years of age (Laverty et al., 1991).

Chondrocyte clusters were the most common lesiontypes present in the HAC. We chose to define chondrocyte clusters by the width of the chondron based on reports of light microscopy examination of paraffin-embedded specimens and considered that groups of chondrocytes aligned in columns were normal morphology (Murray et al., 2009). Chondrocyte clusters in the HAC were subdivided into marginal and central chondrocyte clusters to test our subjective impression that chondrocyte clusters were seen in the joint margin regions without signs of other lesions in the specimen. Marginal chondrocyte clusters were consistently the most common HAC lesion-type in all joints, but there was no significant association and at best only a weak to moderate correlation of these with other lesion-types in the centrodistal joint. Additionally, the frequency of marginal chondrocyte clusters was high in both the centrodistal and tarsometatarsal joints, whereas the frequencies of all other articular cartilage lesion-types were moderate to high in the centrodistal joint and low in the tarsometatarsal joint. Since OA of the joints of the distal tarsal region in Icelandic horses is reported to be more common in the centrodistal joint compared to the tarsometatarsal joint (Eksell et al., 1999; Björnsdottir et al., $2000 \mathrm{~b}$ ) this is further evidence that marginal chondrocyte clusters were present for reasons other than early OA.

Significant associations and strong correlations were detected between HAC chondrocyte loss, HAC central chondrocyte clusters, ACC arrest and ACC advancement. Additionally, regions of adjacent HAC chondrocyte loss and HAC central chondrocyte clusters were common in our material. We speculate that this pattern is a result of a focal area of severe HAC damage that caused chondrocyte death, surrounded by a region of milder HAC damage that caused chondrocyte injury with a subsequent hypertrophic response of the chondrocytes. A similar spatial pattern was reflected in the ACC arrest and advancement lesions with the ACC arrest usually located deep to the HAC chondrocyte loss and the ACC advancement usually located deep to the HAC chondrocyte clusters. Thus our findings suggest that HAC and ACC damage occurred simultaneously, and raises the possibility that HAC damage resulted in changes of ACC development, which include in some areas thinning or loss of the ACC and in others activation of the mineralisation of HAC and subsequent advancement of the ACC. Links exist between chondrocyte differentiation in the local domain and the rate of extracellular matrix mineralisation (Newton et al., 2012). Chondrocyte clusters promote hyaline cartilage calcification (Fuerst et al., 2009; van der Kraan and van den Berg, 2012). Thus, advancement of the MF in a region of HAC chondrocyte clusters is likely. Alternatively, a loss of HAC chondrocytes could contribute to focal regions of arrest of the MF. These lesion patterns support our hypothesis that the early morphological changes of lowmotion joint equine OA start in both the HAC and ACC.

ACC might have a role as a selective barrier between the intra-articular and SCB compartments and if the ACC becomes breached by cracks or vascular channels, thin or resorbed then it may be possible that substances can more readily pass between these compartments (Pan et al., 2009; Pan et al., 2012; Suri and Walsh, 2012). Inflammatory substances released by osteoblasts, including cytokines and growth factors, have been shown to result in changes in chondrocyte metabolism and contribute to cartilage degradation (Sanchez et al., 2005a; Sanchez et al., 2005b). ACC also plays a role in dissipating forces at the bone cartilage interface (Oegema et al., 1997) and thus if ACC is thin or damaged the risk of SCB injury increases. This means a thin or damaged ACC both increases the risk of SCB injury and increases the exposure of HAC to inflammatory substances that are released by injured SCB. The combination may result in initiation and propagation of OA in the joint. Thus it seems very likely that the thinning, loss or damage of ACC and its barrier integrity could be a key event in the development of OA.

General theories of OA pathogenesis emphasise advancement of the ACC and acceleration of endochondral ossification in the SCB (Burr, 2005; Brandt et al., 2009), but we have identified an additional process that we believe is caused by arrest or slowing of the ACC advance. The different lesion patterns are likely to be a result of the low-motion and high-compression loading of the joints of the equine distal tarsal region, which contrast to more commonly studied high-motion, dynamically loaded joints such as the equine metacarpophalangeal joint and the human knee. This is supported by a study of the equine carpus that shows variations in loading affect the type of osteochondral abnormalities that develop (Kim et al., 2012). Additionally much of the knowledge about the development and morphology of OA results from studies 
of experimentally induced OA in animals and studies of advanced OA in human joints (Poole et al., 2010; Heinegard and Saxne, 2011). The Icelandic horse tarsal joints in our study are an example of the early stages of natural OA development. Studies of this type of material are unusual and it is possible that we have identified a stage of natural OA development that has not previously been well documented. The lesion patterns we detected are also likely to be related to the relatively young age of the horses in the study group. Appendicular bone growth in Icelandic horses is complete when they are approximately three years of age (Strand et al., 2007). Thus the horses in our study are very close to but perhaps not completely skeletally mature. It is likely that the ACC arrest lesions have developed during the months prior to slaughter when endochondral ossification would still have been active. During this period the ACC would have been advancing rapidly, and damage to the hypertrophic chondrocytes involved in growth and the formation of mineralised cartilage at this time could have a major effect on the ACC thickness and MF contour.

Contrary to our descriptions of ACC arrest, it has been found in the distal metacarpal condyle of young Thoroughbred horses using calcein labels that the areas with the most rapid advance of the ACC were regions with thinner ACC with relatively higher mineral density compared to areas of slower advance (Doube et al., 2007). There are several reasons why we believe that the ACC arrest regions in our specimens do not follow the pattern described by Doube et al. (2007). The level of the MF in areas of ACC arrest was always lagging behind adjacent areas of normal or increased thickness ACC, indicating a slowing of the advance of the ACC in these areas. The areas of most rapid ACC advancement in the study of distal metacarpal condyles were detected in areas of relatively low loading, whereas the ACC arrest lesions we detected were often located within 0.5 to $2 \mathrm{~mm}$ from the dorsal, lateral or medial joint margins in regions of the joint where loading is likely to be high (Murray et al., 2007). Future studies of the joints of the distal tarsal region in young Icelandic horses using calcein labels would be valuable to test the interpretation of our morphological findings and further investigate the behaviour of the ACC in early OA.

In clinical radiology involving $\mathrm{CT}$ or MRI studies of OA in the joints of the equine distal tarsal region, various names have been given to concave irregularities in the articular aspect of the mineralised tissues including SCB defects, SCB plate irregularities, osteochondral junction defects, and articular mineralisation front defects (Laverty et al., 1991; Björnsdottir et al., 2004; Branch et al., 2007; Ley et al., 2013). These clinical imaging methods are not able to differentiate the ACC from the SCB, so even though the $\mathrm{SCB}$ is most often implicated as the tissue involved in the defect it is likely that some of these concave irregularities are areas of ACC arrest.

Osteophytic outgrowths in the central portion of the articular space detected with MRI are termed 'central osteophytes' (McCauley et al., 2001). These lesions are described from CT, MRI and light microscopy studies of the equine joints (Olive et al., 2009; Ley et al., 2013). In the centrodistal joint of Icelandic horses these lesions were described as a bulging MF formed of ACC and SCB and when detected in MRI images were significantly associated with OA detected by light microscopy (Ley et al., 2013). In the metacarpophalangeal joints of adult horses, light microscopy of paraffin-embedded specimens showed these lesions to be projections of dense SCB that disrupt the cartilage (Olive et al., 2009). We did not identify SCB projections similar to those described by Olive et al. (2009), possibly due to the different ages and loading history of the two study groups, but we did observe that the contour of the cement line approximately followed the contour of the MF. In other words, in regions of ACC arrest the ossifying front of the SCB was usually lagging behind, and in regions of ACC advance the ossifying front of the SCB was usually further advanced compared to areas where there were no changes detected in the ACC (Figs. 4d, 5d, 6b, 8d). We considered that the contour of the cement line was varying due to the changes occurring in the ACC and that the rate of ACC advance was influencing the rate of SCB advance. In clinical CT or MRI images the appearance of areas we have described as ACC advancement would be expected to have the appearance corresponding to the existing descriptions of central osteophytes. In a morphological context, ACC advancement is not an outgrowth of bone and thus the term osteophyte is misleading. We recommend the use of the term MF advancement to describe this lesion when the ACC and SCB tissues cannot be defined, or if the tissues can be defined ACC or SCB advancement as appropriate.

This is the first report of HIP protrusions from the MF in the joints of the distal tarsal region of horses. Corresponding lesions are described in equine metacarpo/ metatarsophalangeal joints, where filling of traumatic ACC cracks and communicating cavities in the HAC with extracellular fluid or plasma that subsequently calcifies is proposed as the cause of the lesions (Boyde, 2003; Boyde and Firth, 2008; Boyde et al., 2011; Whitton et al., 2013). The identification of this lesion-type provides an explanation for a feature described as intra-articular and chondral mineralisations in CT images of centrodistal joints from a study performed on the contralateral centrodistal joints of the same horses as used in the current study (Ley et al., 2013). Although an uncommon lesion-type, HIP protrusions from the MF were always associated with other HAC and ACC lesion-types and even though statistical associations were not detected in the centrodistal joint, we still consider this lesion to be associated with other HAC and ACC lesion-types. On the other hand, associations between HIP within ACC and the other HAC and ACC lesion-types were not obvious, since HIP within ACC was seen in joints that did not show other changes.

Lesions of the SCB were rare in the distal tarsal joints. Resorption of the SCB was the only lesion-type we were able to identify and this lesion-type had a low frequency. BSE SEM of PMMA-embedded blocks is a proven method for assessing the morphology and mineral density of the SCB in horses (Boyde, 2003; Boyde and Firth, 2005; Boyde and Firth, 2008), so if other SCB lesion-types were present these changes should have been readily detectable. It appears that SCB lesions are rare in the early stages of distal tarsal OA. However, when specimens that included SCB resorption were examined individually it was found that all SCB resorption lesions occurred together with other 
ACC and HAC lesion-types (Fig. 6b). Thus we consider this lesion to have an association with other ACC and HAC lesion-types.

Lesion-types of the joint margins were divided into margin extension and margin erosion, but both these lesion-types resulted in a similar peaked to spur shaped joint margin, which is the typical shape of a 'marginal osteophyte' seen by radiology, CT and MRI. Surprisingly, there were no statistically significant associations between the margin lesion-types and any of the other lesion-types. Both of the joint margin lesion-types had a high frequency in PMMA-embedded centrodistal and tarsometatarsal joint specimens and it was notable that margin erosions were detected in all macerated specimens from the centrodistal and tarsometatarsal joints. These results suggest that the processes of joint margin bone formation and erosion are independent of each other and independent of the lesions of the HAC and ACC.

A weakness of our evaluation of associations between lesion-types was that we collected data from joint specimens of varying size and location. This meant that when lesion-types were located somewhat distant from each other but still within the examined joint they were considered equal to close and adjacent lesion-types. Thus the associations we have reported are at a joint region specimen level. We have attempted to compensate for this weakness in our lesion-type descriptions and figures, where we include information about descriptive lesion associations observed in the specimens.

\section{Conclusions}

Our results indicate that osteochondral lesion-types with features of osteoarthritis have a high frequency in the centrodistal joints of young Icelandic horses and that the early morphological changes of osteoarthritis occur in the hyaline and calcified articular cartilage, rather than in the subchondral bone. We speculate that chondrocyte death and chondrocyte hyperplasia in the hyaline articular cartilage influence the development of calcified articular cartilage arrest and advancement, respectively. Thinning or loss of the calcified articular cartilage resulting from arrest lesion-types may have a key role in the development and perpetuation of osteoarthritis in the low-motion centrodistal joint, since these changes have the potential to result in areas in the calcified articular cartilage that allow increased transfer of substances between the subchondral bone and the intra-articular compartments. Young Icelandic horses offer a promising model for the study of calcified articular cartilage behaviour in early osteoarthritis of low-motion joints, a morphological region that we believe is crucial in the early stages of osteoarthritis development.

\section{Acknowledgements}

This work was supported by funding grants from the Royal Swedish Academy of Agriculture and Forestry (H10-0265-CFH), the Swedish Norwegian Foundation for
Equine Research (H0847237), and the SLU travel fund. The authors would like to acknowledge Maureen Arora for preparation of the specimens for BSE SEM and CSLM, Beate Hillman, Hans Kanbjer, Lars Hammarsten and Johan Karevik for assistance with sawing of tarsal joints, and Claudia von Brömssen for statistical assistance. We wish to confirm that there are no known conflicts of interest associated with this publication and there has been no significant financial support for this work that could have influenced its outcome.

\section{References}

Arnason T, Björnsdóttir S (2003) Heritability of ageat-onset of bone spavin in Icelandic horses estimated by survival analysis. Livest Prod Sci 79: 285-293.

Axelsson M, Bjornsdottir S, Eksell P, Haggstrom J, Sigurdsson H, Carlsten J (2001) Risk factors associated with hindlimb lameness and degenerative joint disease in the distal tarsus of Icelandic horses. Equine Vet J 33: 84-90.

Barneveld A, van Weeren PR (1999) Early changes in the distal intertarsal joint of Dutch Warmblood foals and the influence of exercise on bone density in the third tarsal bone. Equine Vet J Suppl. 31: 67-73.

Batiste DL, Kirkley A, Laverty S, Thain LMF, Spouge AR, Gati JS, Foster PJ, Holdsworth DW (2004) Highresolution MRI and micro-CT in an ex vivo rabbit anterior cruciate ligament transection model of osteoarthritis. Osteoarthritis Cartilage 12: 614-626.

Benjamini Y, Hochberg Y (1995) Controlling the False Discovery Rate - a Practical and Powerful Approach to Multiple Testing. J Roy Stat Soc B Met 57: 289-300.

Bittersohl B, Mamisch TC, Welsch GH, Stratmann J, Forst R, Swoboda B, Bautz W, von Rechenberg B, Cavallaro A (2009) Experimental model to evaluate in vivo and in vitro cartilage MR imaging by means of histological analyses. Eur J Radiol 70: 561-569.

Björnsdottir S, Arnason T, Axelsson M, Eksell P, Sigurðsson H, Carlsten J (2000a) The heritability of degenerative joint disease in the distal tarsal joints in Icelandic horses. Livest Prod Sci 63: 77-83.

Björnsdottir S, Axelsson M, Eksell P, Sigurðsson H, Carlsten J (2000b) Radiographic and clinical survey of degenerative joint disease in the distal tarsal joints in Icelandic horses. Equine Vet J 32: 268-272.

Björnsdottir S, Ekman S, Eksell P, Lord P (2004) High detail radiography and histology of the centrodistal tarsal joint of young Icelandic horses. Equine Vet J 36: 5-11.

Boyde A (2003) The real response of bone to exercise. J Anat 203: 173-189.

Boyde A (2012a) Scanning electron microscopy of bone. In: Helfrich MH, Ralson SH (eds) Bone Research Protocols, Springer, New York, pp 365-400.

Boyde A (2012b) Staining plastic blocks with triiodide to image cells and soft tissues in backscattered electron SEM of skeletal and dental tissues. Eur Cell Mater 24: 154-160.

Boyde A (2013) Joint histology by iodine staining. J Anat 223: 84. 
Boyde A, Firth EC (2004) Articular calcified cartilage canals in the third metacarpal bone of 2-year-old thoroughbred racehorses. J Anat 205: 491-500.

Boyde A, Firth EC (2005) Musculoskeletal responses of 2-year-old Thoroughbred horses to early training. 8. Quantitative back-scattered electron scanning electron microscopy and confocal fluorescence microscopy of the epiphysis of the third metacarpal bone. N Z Vet J 53: 123 132.

Boyde A, Firth EC (2008) High resolution microscopic survey of third metacarpal articular calcified cartilage and subchondral bone in the juvenile horse: possible implications in chondro-osseous disease. Microsc Res Tech 71: 477-488.

Boyde A, Lovicar L, Zamecnik J (2005) Combining confocal and BSE SEM imaging for bone block surfaces. Eur Cell Mater 9: 33-38.

Boyde A, Riggs CM, Bushby AJ, McDermott B, Pinchbeck GL, Clegg PD (2011) Cartilage damage involving extrusion of mineralisable matrix from the articular calcified cartilage and subchondral bone. Eur Cell Mater 21: 470-478.

Branch MV, Murray RC, Dyson SJ, Goodship AE (2007) Magnetic resonance imaging of the equine tarsus. Clin Tech Equine Pract 6: 96-102.

Brandt KD, Radin EL, Dieppe PA, van de Putte L (2006) Yet more evidence that osteoarthritis is not a cartilage disease. Ann Rheum Dis 65: 1261-1264.

Brandt KD, Dieppe P, Radin E (2009) Etiopathogenesis of osteoarthritis. Med Clin North Am 93: 1-24.

Burr DB (2005) Increased biological activity of subchondral mineralized tissues underlies the progressive deterioration of articular cartilage in osteoarthritis. J Rheumatol 32: 1156-1158.

Byam-Cook KL, Singer ER (2009) Is there a relationship between clinical presentation, diagnostic and radiographic findings and outcome in horses with osteoarthritis of the small tarsal joints? Equine Vet J 41: 118-123.

Calvo E, Palacios I, Delgado E, Sánchez-Pernaute O, Largo R, Egido J, Herrero-Beaumont G (2004) Histopathological correlation of cartilage swelling detected by magnetic resonance imaging in early experimental osteoarthritis. Osteoarthritis Cartilage 12: 878-886.

Dieppe P, Kirwan J (1994) The localization of osteoarthritis. Br J Rheumatol 33: 201-203.

Doube M, Firth EC, Boyde A (2007) Variations in articular calcified cartilage by site and exercise in the 18-month-old equine distal metacarpal condyle. Osteoarthritis Cartilage 15: 1283-1292.

Eksell P, Axelsson M, Brostrom H, Roneus B, Haggstrom J, Carlsten J (1998) Prevalence and risk factors of bone spavin in Icelandic horses in Sweden: a radiographic field study. Acta Vet Scand 39: 339-348.

Eksell P, Uhlhorn H, Carlsten J (1999) Evaluation of different projections for radiographic detection of tarsal degenerative joint disease in Icelandic Horses. Vet Radiol Ultrasound 40: 228-232.

Fairburn A, Dyson S, Murray R (2010) Clinical significance of osseous spurs on the dorsoproximal aspect of the third metatarsal bone. Equine Vet J 42: 591-599.
Fuerst M, Bertrand J, Lammers L, Dreier R, Echtermeyer F, Nitschke Y, Rutsch F, Schafer FK, Niggemeyer O, Steinhagen J, Lohmann CH, Pap T, Ruther W (2009) Calcification of articular cartilage in human osteoarthritis. Arthritis Rheum 60: 2694-2703.

Glowatzki-Mullis ML, Muntwyler J, Pfister W, Marti E, Rieder S, Poncet PA, Gaillard C (2006) Genetic diversity among horse populations with a special focus on the Franches-Montagnes breed. Anim Genet 37: 33-39.

Guermazi A, Zaim S, Taouli B, Miaux Y, Peterfy CG, Genant HG (2003) MR findings in knee osteoarthritis. Eur Radiol 13: 1370-1386.

Heinegard D, Saxne T (2011) The role of the cartilage matrix in osteoarthritis. Nature reviews. Rheumatology 7: 50-56.

Kim W, Kawcak CE, McIlwraith CW, Firth EC, Broom ND (2012) Histologic and histomorphometric evaluation of midcarpal joint defects in Thoroughbreds raised with and without early conditioning exercise. Am J Vet Res 73: 498-507.

Labens R, Mellor DJ, Voute LC (2007) Retrospective study of the effect of intra-articular treatment of osteoarthritis of the distal tarsal joints in 51 horses. Vet Rec 161: 611-616.

Lanovaz JL, Khumsap S, Clayton HM, Stick JA, Brown $J$ (2002) Three-dimensional kinematics of the tarsal joint at the trot. Equine Vet J Supplement 34: 308-313.

Laverty S, Stover SM, Belanger D, O'Brien TR, Pool RR, Pascoe JR, Taylor K, Harrington T (1991) Radiographic, high detail radiographic, microangiographic and histological findings of the distal portion of the tarsus in weanling, young and adult horses. Equine Vet J 23: 413-421.

Ley CJ, Ekman S, Dahlberg LE, Björnsdóttir S, Hansson K (2013) Evaluation of osteochondral sample collection guided by computed tomography and magnetic resonance imaging for early detection of osteoarthritis in centrodistal joints of young Icelandic horses. Am J Vet Res 74: 874-887.

Mankin HJ, Lippiello L (1970) Biochemical and metabolic abnormalities in articular cartilage from osteoarthritic human hips. J Bone Joint Surg Am 52: 424-434.

McCauley TR, Kornaat PR, Jee WH (2001) Central osteophytes in the knee: prevalence and association with cartilage defects on MR imaging. Am J Roentgenol 176: 359-364.

McGibbon CA, Trahan CA (2003) Measurement accuracy of focal cartilage defects from MRI and correlation of MRI graded lesions with histology: a preliminary study. Osteoarthritis Cartilage 11: 483-493.

Mcllwraith CW, Frisbie DD, Kawcak CE, Fuller CJ, Hurtig M, Cruz A (2010) The OARSI histopathology initiative - recommendations for histological assessments of osteoarthritis in the horse. Osteoarthritis Cartilage 18 Supplement 3: S93-S105.

Murray RC, Blunden TS, Schramme MC, Dyson SJ (2006) How does magnetic resonance imaging represent histologic findings in the equine digit? Vet Radiol Ultrasound 47: 17-31.

Murray RC, Branch MV, Dyson SJ, Parkin TDH, Goodship AE (2007) How does exercise intensity and type 
affect equine distal tarsal subchondral bone thickness? J Appl Physiol 102: 2194-2200.

Murray RC, Blunden TS, Branch MV, Tranquille CA, Dyson SJ, Parkin TD, Goodship AE (2009) Evaluation of age-related changes in the structure of the equine tarsometatarsal osteochondral unit. Am J Vet Res 70: 30-36.

Newton PT, Staines KA, Spevak L, Boskey AL, Teixeira CC, Macrae VE, Canfield AE, Farquharson C (2012) Chondrogenic ATDC5 cells: an optimised model for rapid and physiological matrix mineralisation. Int $\mathrm{J}$ Mol Med 30: 1187-1193.

Oegema TR, Jr., Carpenter RJ, Hofmeister F, Thompson RC, Jr. (1997) The interaction of the zone of calcified cartilage and subchondral bone in osteoarthritis. Microsc Res Tech 37: 324-332.

Olive J, D'Anjou M-A, Girard C, Laverty S, Theoret CL (2009) Imaging and histological features of central subchondral osteophytes in racehorses with metacarpophalangeal joint osteoarthritis. Equine Vet J 41: 859-864.

Olive J, D'Anjou M, Girard C, Laverty S, Theoret C (2010) Fat-suppressed spoiled gradient-recalled imaging of equine metacarpophalangeal articular cartilage. Vet Radiol Ultrasound 51: 107-115.

Pan J, Zhou X, Li W, Novotny JE, Doty SB, Wang L (2009) In situ measurement of transport between subchondral bone and articular cartilage. J Orthop Res 27: 1347-1352.

Pan J, Wang B, Li W, Zhou X, Scherr T, Yang Y, Price C, Wang L (2012) Elevated cross-talk between subchondral bone and cartilage in osteoarthritic joints. Bone 51: 212217.

Peterfy CG, Guermazi A, Zaim S, Tirman PFJ, Miaux Y, White D, Kothari M, Lu Y, Fye K, Zhao S, Genant HK (2004) Whole-Organ Magnetic Resonance Imaging Score (WORMS) of the knee in osteoarthritis. Osteoarthritis Cartilage 12: 177-190.

Pool RR (1996) Pathological manifestations of joint disease in the athletic horse. In: McIlwraith CW, Trotter GW (eds) Joint Disease in the Horse, Saunders, Philadelphia, pp 87-104.

Poole R, Blake S, Buschmann M, Goldring S, Laverty S, Lockwood S, Matyas J, McDougall J, Pritzker K, Rudolphi K, van den Berg W, Yaksh T (2010) Recommendations for the use of preclinical models in the study and treatment of osteoarthritis. Osteoarthritis Cartilage 18 Supplement 3: S10-S16.

Pritzker KPH, Gay S, Jimenez SA, Ostergaard K, Pelletier JP, Revell PA, Salter D, van den Berg WB (2006) Osteoarthritis cartilage histopathology: grading and staging. Osteoarthritis Cartilage 14: 13-29.

Sanchez C, Deberg MA, Piccardi N, Msika P, Reginster JY, Henrotin YE (2005a) Osteoblasts from the sclerotic subchondral bone downregulate aggrecan but upregulate metalloproteinases expression by chondrocytes. This effect is mimicked by interleukin- $6,-1$ beta and oncostatin M pretreated non-sclerotic osteoblasts. Osteoarthritis Cartilage 13: 979-987.

Sanchez C, Deberg MA, Piccardi N, Msika P, Reginster JY, Henrotin YE (2005b) Subchondral bone osteoblasts induce phenotypic changes in human osteoarthritic chondrocytes. Osteoarthritis Cartilage 13: 988-997.

Scanzello CR, Goldring SR (2012) The role of synovitis in osteoarthritis pathogenesis. Bone 51: 249-257.

Schamhardt HC, Hartman W, Lammertink JL (1989) Forces loading the tarsal joint in the hind limb of the horse, determined from in vivo strain measurements of the third metatarsal bone. Am J Vet Res 50: 728-733.

Shively MJ (1982) Correct anatomic nomenclature for the joints of the equine tarsus. Equine Practice 4: 9-13.

Sisson S (1975) Equine syndesmology. In: Getty R (ed) The Anatomy of the Domestic Animals, Saunders, Philadelphia, pp 349-375.

Squires GR, Okouneff S, Ionescu M, Poole AR (2003) The pathobiology of focal lesion development in aging human articular cartilage and molecular matrix changes characteristic of osteoarthritis. Arthritis Rheum 48: 12611270 .

Strand E, Braathen LC, Hellsten MC, Huse-Olsen L, Bjornsdottir S (2007) Radiographic closure time of appendicular growth plates in the Icelandic horse. Acta Vet Scand 49: 19.

Suri S, Walsh DA (2012) Osteochondral alterations in osteoarthritis. Bone 51: 204-211.

Tranquille CA, Dyson SJ, Blunden AS, Collins SN, Parkin TDH, Goodship AE, Murray RC (2011) Histopathologic features of distal tarsal joint cartilage and subchondral bone in ridden and pasture-exercised horses. Am J Vet Res 72: 33-41.

van der Kraan PM, van den Berg WB (2012) Chondrocyte hypertrophy and osteoarthritis: role in initiation and progression of cartilage degeneration? Osteoarthritis Cartilage 20: 223-232.

Veje K, Hyllested-Winge JL, Ostergaard K (2003) Topographic and zonal distribution of tenascin in human articular cartilage from femoral heads: normal versus mild and severe osteoarthritis. Osteoarthritis Cartilage 11: 217 227.

Waldschmidt JG, Braunstein EM, Buckwalter KA (1999) Magnetic resonance imaging of osteoarthritis. Rheum Dis Clin North Am 25: 451-465.

Watrous BJ, Hultgren BD, Wagner PC (1991) Osteochondrosis and juvenile spavin in equids. Am J Vet Res 52: 607-612.

Whitton RC, Mirams M, Mackie EJ, Anderson GA, Seeman E (2013) Exercise-induced inhibition of remodelling is focally offset with fatigue fracture in racehorses. Osteoporos Int 24: 2043-2048.

\section{Discussion with Reviewers}

Reviewer I: In some of the specimens there may have been a three day lapse between harvest and processing for microscopy. This is a challenge facing researchers undertaking similar comparative imaging studies. How could this delay affect cell preservation and the reliable assessment of chondrocyte density, or, more importantly, chondrocyte necrosis? The authors should address how they were sure it was chondrocyte necrosis due to OA and not 
due to the delay or artefacts due to processing. What were the visual criteria to determine the presence of chondrocyte necrosis? Standard histology with light microscopy permits a good examination of cell morphology for these purposes. The absence of chondrocytes in the lacunae could be real, secondary to delay in processing or an artefact.

Authors: There are several reasons we believe the regions of chondrocyte loss that we observed were not due to the delay to processing or processing artefacts and were due to the chondrocyte necrosis that occurs in OA.

The regions of chondrocyte loss we identified were focal in all cases. We did not have any specimens where a generalised distribution of chondrocyte loss was present. If chondrocyte loss was caused by autolysis or processing artefacts then generalised changes would be expected.

The correlations and associations we detected between HAC chondrocyte loss, HAC central chondrocyte clusters and $\mathrm{ACC}$ arrest were very strong and further strong associations were detected between HAC chondrocyte loss, HAC fibrillation and ACC advancement. Thus chondrocyte loss is associated and correlated with other microscopic features of OA, strongly suggesting that the chondrocyte loss we have detected is due to OA related necrosis.

We used the contralateral tarsal joints from the same horses as were used in this manuscript in a light microscopy study of the centrodistal joints (Ley et al., 2013). The delay to processing was identical in both studies. Multiple paraffin-embedded specimens were taken from the right centrodistal joints. Using light microscopy we observed necrosis of the chondrocytes (eosinophilic cytoplasm and loss of nuclei in H\&E stain and loss of toluidine blue staining in adjacent extra-cellular matrix) in regions that correspond to those where cell loss was detected in the specimens used for the current manuscript.

The joints in the current study remained unopened and intact up until the time that they were manually sectioned with a band saw and immediately after this sawing the osteochondral specimens were placed in formalin or frozen. A study of bovine articular cartilage shows that chondrocytes in refrigerated joint specimens maintained cellular metabolic activity and remained viable for five days after slaughter (Schachar et al., 1994), supporting that processing artefacts should be minimal with the 45 to $69 \mathrm{~h}$ delay time to fixation/freezing of our joint specimens. Additionally, a study of bone tissue found that all bone cells have good viability in vitro, surviving chilling for many days (Vesely et al., 1992).

Reviewer I: The marginal changes are very interesting. Could the authors speculate a little more on their meaning? Authors: Marginal erosions and extensions were common in the centrodistal and tarsometatarsal joints and the lack of association of these lesions with the articular cartilage lesions in the centrodistal joints suggests that these lesions have additional or alternative causes compared to the articular cartilage lesion-types. Using our methods to analyse lesion-types we only investigate associations between lesions that are present in early OA and not present in normal joints. We do not investigate associations between lesion-types that are small in normal joints but larger and more widespread (within a joint) in OA. Thus with our study design we cannot exclude that marginal erosions and extensions have a role in early OA.

The marginal erosions are perhaps part of the normal process of bone modelling and/or remodelling in these joints, and in this case this change may be occurring constantly on the joint margins. The horses used in the study are young so it is possible that the high frequency of marginal erosions is related to the final stages of growth modelling and mineralisation of the central and third tarsal bones.

We observed that marginal extensions occurred due to mineralisation of several tissue types (fibrous joint capsule, periosteum, fibrocartilage), which raises the possibility of several different causes ranging from adaptive changes in joint shape to increased cellular activity in OA that produce the same outcome - a marginal osteophyte. This complicates the interpretation of the cause and meaning of marginal extensions. We did not attempt to group marginal extensions according to the tissue type undergoing mineralisation due to the complexities created by simultaneous mineralisation of multiple tissue types within a marginal extension and large variations in the stage of development of the marginal extensions. Joints are constantly modelling shape and form in response to loading, age and disease (Bullough, 2004) and margin extensions are likely to represent a part of this multifactorial process.

Reviewer I: Can the authors speculate as to why fewer lesions are encountered in the talocalcaneal-centroquartal joints?

Authors: Without knowing the cause of the moderate to high frequencies of the centrodistal and tarsometatarsal joint osteochondral lesions, it is challenging to speculate why the talocalcaneal-centroquartal joints have fewer lesions. It is suspected that distal tarsal OA is a mechanically induced injury (Eksell et al., 1998; Björnsdottir et al., 2004; Fairburn et al., 2010) and in the Icelandic horse poor joint conformation resulting in an uneven distribution of the mechanical forces in the joints has been proposed to be a cause (Eksell et al., 1998; Axelsson et al., 2001; Björnsdottir et al., 2004). If these theories are correct then factors that affect the distribution and dissipation of forces on the joint surface, such as joint surface area, joint surface contour, articular cartilage and subchondral bone volume and composition, and joint motion during weight bearing, might all influence the osteochondral lesion incidence. Studies that investigate differences between these features in the equine distal tarsal joints are lacking, although 3D imaging techniques such as CT and MRI have now reached a stage where they can be used to examine volumes, surface areas and shapes of entire joint regions in detail, which may help to answer some of these questions.

Reviewer I: Can the authors speculate as to why these initiating causes and changes arise in young animals, not in training or racing? Could they be related to earlier developmental problems?

Authors: We are not aware of any developmental problems in this group of horses that could have resulted in the osteochondral lesions that we have detected. Although osteochondrosis has been reported to be the 
cause of osteochondral lesions in the equine distal tarsal region in young horses (Watrous et al., 1991), we did not find morphological evidence of osteochondrosis in our specimens and do not believe it is the cause of the lesions we describe. There is of course individual variation between the leg and joint conformations of the horses in our study and this is an area that we are continuing to investigate.

Reviewer II: The quite selective methodology proved incomplete, surface staining of the same PMMA-blocks or ground sections with toluidine blue or Giemsa-solution would have helped a lot for clarification.

Authors: We are fully aware of these possibilities (Plenk et al., 2008), but in our manuscript point out the utility of a very simple and brand new method for studying intact 'histomorphology'. To address this, we re-polished the specimen shown in Fig. 6b, stained it with toluidine blue and conducted light microscopy. We then re-polished the same region, stained it with ammonium triiodide and repeated the SEM imaging. We used correlation software (Boyde et al., 2005) to overlap the light microscopy and SEM images and found a good correspondence.

Reviewer II: Could the authors comment on the differences of cellular and ECM details visible in cartilage and bone when comparing the two applications of iodine-staining. Authors: We take it that this question addresses differences between (wet) triiodide ion solution phase and (dry) iodine vapour phase staining techniques. This is a big topic which requires much further research and evaluation. However, in the broadest view, 'things' stain in a similar way with both approaches. Wet staining is rapid, and depending on the stain concentration, can be done in one to thirty minutes. The stain can be applied as drops to only parts of the block surface. It is difficult to avoid the retention of stain solution in block surface defects whilst washing off the block before drying. Dry staining is slow, and might typically last three days to three weeks, but one can go back to do some more if the intensity is insufficient.

Reviewer II: What caused the apparent loss of the original contrast and resolution of unstained BSE-SEM images?

Authors: When imaging unstained PMMA-embedded blocks, we set the brightness and contrast values to image only the mineral phase in bone and calcified cartilage. We have exquisite sensitivity to mineral content, which can be evaluated when using suitable standards with a range of electron backscattering coefficients (Boyde and Firth, 2005). When the blocks have iodine added in the superficial layers, the contrast is reduced because the iodine binds to all non-mineralised soft tissue elements, but also because it also binds to the matrix within mineralised regions. Within ACC, there is much less-well-mineralised cartilage matrix. This binds iodine more than the better mineralised matrix, so that, again, the contrast in ACC is reduced after staining.

Reviewer II: Could there also be a difference in the BSEimaging with "conventional" and "environmental" SEM? Authors: BSE SEM images obtained at high vacuum when using a carbon coating to dissipate the charge are in most respects indistinguishable from those obtained in a $50 \mathrm{~Pa}$ chamber pressure with uncoated specimens. However, no quantitation of the backscattered electron signal (as in evaluating mineral content) should be attempted in uncoated specimens. This is because the eradication of charging problems in the uncoated specimen by attracting positive gas ions to the otherwise negatively charged surface varies slightly from field to field depending on the overall mineral content in the field.

\section{Additional References}

Bullough PG (2004) The role of joint architecture in the etiology of arthritis. Osteoarthritis Cartilage 12 Supplement: $2-9$.

Plenk H, Jr., Shum JC, Cruise GM, Killer M (2008) Cartilage and bone neoformation in rabbit carotid bifurcation aneurysms after endovascular coil embolization. Eur Cell Mater 16: 69-79.

Schachar NS, Cucheran DJ, McGann LE, Novak KA, Frank CB (1994) Metabolic activity of bovine articular cartilage during refrigerated storage. J Orthop Res 12: 15-20.

Vesely P, Boyde A, Jones SJ (1992) Behaviour of osteoclasts in vitro: contact behaviour of osteoclasts with osteoblast-like cells and networking of osteoclasts for 3D orientation. J Anat 181: 277-291. 